\title{
Taxonomic Studies on Some Members of the Genus Abutilon Mill. (Malvaceae)
}

\author{
Dhafer A. Alzahrani1 ${ }^{*}$, Enas J. Albokhari ${ }^{1,2}$, Abrar Khoj ${ }^{1}$ \\ ${ }^{1}$ Department of Biological Sciences, Faculty of Sciences, King Abdulaziz University, Jeddah, Saudi Arabia \\ ${ }^{2}$ Department of Biological Sciences, Faculty of Applied Sciences, Umm Al-Qura University, Makkah, Saudi Arabia \\ Email: ^dalzahrani@kau.edu.sa, *dhaferalzahrani@hotmail.com
}

How to cite this paper: Alzahrani, D.A. Albokhari, E.J. and Khoj, A. (2021) Taxonomic Studies on Some Members of the Genus Abutilon Mill. (Malvaceae). American Journal of Plant Sciences, 12, 199-220. https://doi.org/10.4236/ajps.2021.122012

Received: January 10, 2021

Accepted: February 23, 2021

Published: February 26, 2021

Copyright (c) 2021 by author(s) and Scientific Research Publishing Inc. This work is licensed under the Creative Commons Attribution International License (CC BY 4.0).

http://creativecommons.org/licenses/by/4.0/

(c) (i) Open Access

\begin{abstract}
The relationship between six Abutilon species was examined using different taxonomic investigation tools. The investigation was carried out using morphological and numerical studies. Fresh materials of Abutilon species were collected from different localities in Saudi Arabia during 2018 and 2019. Numerical analysis was based on the Principle Coordinates, the Principle Component and the Unweighted Pair Group Method with Arithmetic Algorithm Clustering. The results indicated that there were significant differences based on the morphological characters especially in the leaves, fruits and flowers features. Morphometric studies revealed that the six species of Abutilon clearly separated in all different analysis.
\end{abstract}

\section{Keywords}

Taxonomy, Malvaceae, Abutilon, Morphological Characters, Morphometric Analysis, UPGMA, PCoA, PCA

\section{Introduction}

Malvaceae is one of the flowering plants, which has been divided into nine subfamilies, containing about 244 genera, and 4225 species [1], distributed in tropic and subtropical regions and all over the world. This family includes the economically important plants such as cotton, okra and other ornamental shrubs. Genus Abutilon Mill. belongs to subfamily Malvoideae, Malvaceae family, comprising about 200 species, annual or perennial herbs and shrubs or even small trees, distributed in tropics and subtropics of the Americas, Africa, Asia, and Australia. The genus has significant importance, this is due to the fibbers and they contain different chemical compounds such as flavonoids, sterols, triterpenes, anthocyanins and fatty acids [2] [3]. 
Bentham and Hooker [4] divided Malveae into four subtribes based on the carpel arrangement, the number and position of ovule per carpel: Abutilinae, Malopinae, Malvinae, and Sidinae. While Hutchinson Hutchinson [5] reclassified Malvaceae and its tribes by combined the two subtribes Abutilinae and Sidinae into tribe Abutileae. Fryxell [6] placed Herrisantia, Robinsonella and Sida alliances into the genus Abutilon. The tribes (Abutileae and Malveae) were included in the system of [7]. [8] separated the two subtribes Malveae and Sidinae based on molecular data from Malveae under tribe Sidieae. The only one of $A b$ utilon is poorly understood that belongs to infrageneric classification.

Several scientists have observed different species number in genus Abutilon. Baker [9] mentioned 172 species, Mattei [10] counted 100 species, while Hutchinson [5] and Husain and Baquar [11] recorded more than 400 species in genus Abutilon. Mabberley [12] reported 100 species, whereas Fryxell [13] stated around 160 species in genus Abutilon.

Taxonomic studies have been reported on Abutilon Mill. by several authors. El Naggar [14] studied pollen morphological characters of 21 species of Egyptian Malvaceae including 2 species for Abutilon: (Abutilon theophrasti Medik. and $A$. pannosum (G. Forst.) Schledt.), while Shaheen, et al. [15] studied pollen morphology for nine species of Abutilon. Taia [2] recorded five species of Abutilon in Saudi Arabia ( $A$. bidentatum Hoch, A. figarium Webb, A. fruticosum Guill \& Perr., A. hirtum Don and $A$. pannosum Schlecht.) based on morphological characters of leaves, flowers and fruits.

Floral characters are important features used to identifying and describe $A b$ utilon plants as many Angiospermae plants. Flowers were described in several characteristics such as: size, stigma, stamens (number of stamens, forming a staminal tube around the style), number and colour of petals and sepals, length of petioles, bracteate, pedicellate, hermaphrodite, pentamerous, axile placentation [16] [17] [18] [19] [20]. Fruits are one of the most important parts that distinctive Abutilon species from each other. Many authors [11] [16] [17] [18] [20] [21] [22] [23] [24] were used fruit characters to distinguish Abutilon species such as shape, size, number of seed and carpels and present or absent of awn in carpels. Therefore, the main objectives of this study are to provide constant and reliable diagnostic characters by using numerical taxonomic methods and morphological characters, and to compare the results obtained from the morphological and morphometric data.

\section{Materials and Methods}

\subsection{Collection and Identification of Plant Materials}

43 samples were collected during 2018 and 2019 from 19 localities of Saudi Arabia (Table 1). For all collection's samples, the type of habits, the height of plants, the latitude and longitude of all sites were recorded. The samples were placed in $70 \%$ ethanol to study the morphological features; the sample number, location, the name of the collector, and the date of collection were reported. The plant 
Table 1. Accessions of Saudi Arabian Abutilon species collected for the morphological studies, including collection number (Col. no.), date of collection, locality and coordinators information. All vouchers were collected by Abrar Khoj and Dhafer Alzahrani.

\begin{tabular}{|c|c|c|c|c|c|c|}
\hline No. & Col. No. & Species name & $\begin{array}{l}\text { Date of } \\
\text { collection }\end{array}$ & Location & \multicolumn{2}{|c|}{ Coordinates } \\
\hline 1 & $\mathrm{~A} 1$ & A. pannosum (Frost. Fil.) Schltdl & $15-8-2018$ & Khulais & $22^{\circ} 7^{\prime} 16^{\prime \prime N}$ & $39^{\circ} 18^{\prime} 32^{\prime \prime} \mathrm{E}$ \\
\hline 2 & A2 & A. pannosum (Frost. Fil.) Schltdl & $15-8-2018$ & Khulais & $22^{\circ} 7^{\prime} 16^{\prime \prime} \mathrm{N}$ & $39^{\circ} 18^{\prime} 32^{\prime \prime} \mathrm{E}$ \\
\hline 3 & A3 & A. pannosum (Frost. Fil.) Schltdl & $15-8-2018$ & Khulais & $22^{\circ} 7^{\prime} 16^{\prime \prime} \mathrm{N}$ & $39^{\circ} 18^{\prime} 32^{\prime \prime} \mathrm{E}$ \\
\hline 4 & A4 & A. pannosum (Frost. Fil.) Schltdl & $15-8-2018$ & Badr Al-Madinah & $23^{\circ} 45^{\prime} 59^{\prime \prime N}$ & $38^{\circ} 48^{\prime} 9^{\prime \prime} \mathrm{E}$ \\
\hline 5 & A5 & A. pannosum (Frost. Fil.) Schltdl & $15-8-2018$ & Badr Al-Madinah & $23^{\circ} 45^{\prime} 59^{\prime \prime} \mathrm{N}$ & $38^{\circ} 48^{\prime} 9^{\prime \prime} \mathrm{E}$ \\
\hline 6 & A6 & A. pannosum (Frost. Fil.) Schltdl & $15-8-2018$ & Badr Al-Madinah & $23^{\circ} 45^{\prime} 59^{\prime \prime} \mathrm{N}$ & $38^{\circ} 48^{\prime} 9^{\prime \prime} \mathrm{E}$ \\
\hline 7 & A7 & A. pannosum (Frost. Fil.) Schltdl & $18-8-2018$ & Jeddah near to Al Estad region & $21^{\circ} 26^{\prime} 23^{\prime \prime} \mathrm{N}$ & $39^{\circ} 15^{\prime} 14^{\prime \prime} \mathrm{E}$ \\
\hline 8 & A8 & A. pannosum (Frost. Fil.) Schltdl & $18-8-2018$ & Jeddah, east of the highway & $21^{\circ} 26^{\prime} 12^{\prime \prime} \mathrm{N}$ & $39^{\circ} 14^{\prime} 57^{\prime \prime} \mathrm{E}$ \\
\hline 9 & A9 & A. pannosum (Frost. Fil.) Schltdl & $18-8-2018$ & Jeddah, east of the highway & $21^{\circ} 26^{\prime} 12^{\prime \prime N}$ & $39^{\circ} 14^{\prime} 57^{\prime \prime} \mathrm{E}$ \\
\hline 10 & A10 & A. pannosum (Frost. Fil.) Schltdl & $18-8-2018$ & Jeddah, Al-Rehab & $21^{\circ} 32^{\prime} 34^{\prime \prime} \mathrm{N}$ & $39^{\circ} 13^{\prime} 41^{\prime \prime} \mathrm{E}$ \\
\hline 11 & A11 & A. pannosum (Frost. Fil.) Schltdl & $18-8-2018$ & Jeddah, Al-Rehab & $21^{\circ} 32^{\prime} 34^{\prime \prime} \mathrm{N}$ & $39^{\circ} 13^{\prime} 41^{\prime \prime} \mathrm{E}$ \\
\hline 12 & $\mathrm{~A} 12$ & A. pannosum (Frost. Fil.) Schltdl & $22-8-2018$ & Al Bahah, Al-Makhwah Aqaba Haznah & $19^{\circ} 46^{\prime} 49^{\prime \prime} \mathrm{N}$ & $41^{\circ} 26^{\prime} 29^{\prime \prime} \mathrm{E}$ \\
\hline 13 & A13 & A. pannosum (Frost. Fil.) Schltdl & $22-8-2018$ & Al Bahah, Al-Makhwah Aqaba Haznah & $19^{\circ} 46^{\prime} 49^{\prime \prime} \mathrm{N}$ & $41^{\circ} 26^{\prime} 29^{\prime \prime} \mathrm{E}$ \\
\hline 14 & A14 & A. pannosum (Frost. Fil.) Schltdl & $22-8-2018$ & Al Bahah, Al-Makhwah Aqaba Haznah & $19^{\circ} 46^{\prime} 49^{\prime \prime} \mathrm{N}$ & $41^{\circ} 26^{\prime} 29^{\prime \prime} \mathrm{E}$ \\
\hline 15 & A 15 & A. pannosum (Frost. Fil.) Schltdl & $22-8-2018$ & Al Bahah, between Al-Makhwah and Al Mudailif & $19^{\circ} 38^{\prime} 19^{\prime \prime} \mathrm{N}$ & $41^{\circ} 20^{\prime} 25^{\prime \prime} \mathrm{E}$ \\
\hline 16 & A16 & A. pannosum (Frost. Fil.) Schltdl & $22-8-2018$ & Al Bahah, between Al-Makhwah and Al Mudailif & $19^{\circ} 38^{\prime} 19^{\prime \prime} \mathrm{N}$ & $41^{\circ} 20^{\prime} 25^{\prime \prime} \mathrm{E}$ \\
\hline 17 & A 17 & A. pannosum (Frost. Fil.) Schltdl & $26-9-2018$ & Jeddah, near to Harazat & $21^{\circ} 31^{\prime} 11^{\prime \prime} \mathrm{N}$ & $39^{\circ} 15^{\prime} 25^{\prime \prime} \mathrm{E}$ \\
\hline 18 & A18 & A. figarianum Webb & $26-9-2018$ & Jeddah, near to Harazat & $21^{\circ} 31^{\prime} 11^{\prime \prime} \mathrm{N}$ & $39^{\circ} 15^{\prime} 25^{\prime \prime} \mathrm{E}$ \\
\hline 19 & A19 & A. pannosum (Frost. Fil.) Schltdl & $26-9-2018$ & Jeddah, near to Harazat & $21^{\circ} 31^{\prime} 11^{\prime \prime} \mathrm{N}$ & $39^{\circ} 15^{\prime} 25^{\prime \prime} \mathrm{E}$ \\
\hline 20 & A20 & A. pannosum (Frost. Fil.) Schltdl & $4-10-2018$ & Makkah, Al Fayhaa & $21^{\circ} 26^{\prime} 24^{\prime \prime} \mathrm{N}$ & $39^{\circ} 46^{\prime} 1 " \mathrm{E}$ \\
\hline 21 & $\mathrm{~A} 21$ & A. pannosum (Frost. Fil.) Schltdl & $4-10-2018$ & Makkah, Al Awali & $21^{\circ} 21^{\prime} 3 " \mathrm{~N}$ & $39^{\circ} 52^{\prime} 35^{\prime \prime} \mathrm{E}$ \\
\hline 22 & $\mathrm{~A} 22$ & A. pannosum (Frost. Fil.) Schltdl & $4-10-2018$ & Makkah, Al Awali & $21^{\circ} 21^{\prime} 3^{\prime \prime N}$ & $39^{\circ} 52^{\prime} 35^{\prime \prime} \mathrm{E}$ \\
\hline 23 & A23 & A. pannosum (Frost. Fil.) Schltdl & $4-10-2018$ & Makkah, Al Awali & $21^{\circ} 21^{\prime} 3^{\prime \prime} \mathrm{N}$ & $39^{\circ} 52^{\prime} 35^{\prime \prime} \mathrm{E}$ \\
\hline 24 & A24 & A. pannosum (Frost. Fil.) Schltdl & $5-10-2018$ & Makkah Wadi Noman & $21^{\circ} 20^{\prime} 59^{\prime \prime} \mathrm{N}$ & $40^{\circ} 6^{\prime} 25^{\prime \prime} \mathrm{E}$ \\
\hline 25 & A25 & A. pannosum (Frost. Fil.) Schltdl & $5-10-2018$ & Makkah, Wadi Noman & $21^{\circ} 20^{\prime} 59^{\prime \prime} \mathrm{N}$ & $40^{\circ} 65^{\prime \prime} \mathrm{E}$ \\
\hline 26 & A 26 & A. pannosum (Frost. Fil.) Schltdl & $9-10-2019$ & Abha, Aqabat Al Samma & $18^{\circ} 21^{\prime} 12^{\prime \prime} \mathrm{N}$ & $42^{\circ} 8^{\prime} 9^{\prime \prime} \mathrm{E}$ \\
\hline 27 & A27 & A. figarianum Webb & $9-10-2019$ & Abha, Aqabat Al Samma & $18^{\circ} 21^{\prime} 12^{\prime \prime} \mathrm{N}$ & $42^{\circ} 8^{\prime} 9^{\prime \prime} \mathrm{E}$ \\
\hline 28 & A28 & A. pannosum (Frost. Fil.) Schltdl & $9-10-2019$ & Abha, Aqabat Al Samma & $18^{\circ} 21^{\prime} 12^{\prime \prime} \mathrm{N}$ & $42^{\circ} 8^{\prime} 9^{\prime \prime} \mathrm{E}$ \\
\hline 29 & A29 & A. grandifolium (Willd.) Sweet & $9-10-2019$ & Abha, Aqabat Al Samma & $18^{\circ} 21^{\prime} 12^{\prime \prime} \mathrm{N}$ & $42^{\circ} 8^{\prime} 9^{\prime \prime} \mathrm{E}$ \\
\hline 30 & $\mathrm{~A} 30$ & A. grandifolium (Willd.) Sweet & $9-10-2019$ & Abha, Aqabat Al Samma & $18^{\circ} 21^{\prime} 12^{\prime \prime} \mathrm{N}$ & $42^{\circ} 8^{\prime} 9^{\prime \prime} \mathrm{E}$ \\
\hline 31 & A 31 & A. grandifolium (Willd.) Sweet & $9-10-2019$ & Abha, Aqabat Al Samma & $18^{\circ} 21^{\prime} 12^{\prime \prime} \mathrm{N}$ & $42^{\circ} 8^{\prime} 9^{\prime \prime} \mathrm{E}$ \\
\hline 32 & A32 & A. pannosum (Frost. Fil.) Schltdl & $9-10-2019$ & Abha, Aqabat Dhela' & $17^{\circ} 55^{\prime} 13^{\prime \prime N}$ & $42^{\circ} 27^{\prime} 10^{\prime \prime} \mathrm{E}$ \\
\hline 33 & A33 & A. figarianum Webb & $9-10-2019$ & Abha Aqabat Dhela' & $17^{\circ} 55^{\prime} 13^{\prime \prime} \mathrm{N}$ & $42^{\circ} 27^{\prime} 10^{\prime \prime} \mathrm{E}$ \\
\hline 34 & A35 & A. pannosum (Frost. Fil.) Schltdl & $30-10-2019$ & Al Qunfudhah Khamis Harb & $19^{\circ} 10^{\prime} 45^{\prime \prime} \mathrm{N}$ & $41^{\circ} 31^{\prime} 51^{\prime \prime} \mathrm{E}$ \\
\hline 35 & A36 & A. pannosum (Frost. Fil.) Schltdl & $30-10-2019$ & Al Qunfudhah Khamis Harb & $19^{\circ} 10^{\prime} 45^{\prime \prime} \mathrm{N}$ & $41^{\circ} 31^{\prime} 51^{\prime \prime} \mathrm{E}$ \\
\hline
\end{tabular}




\section{Continued}

\begin{tabular}{|c|c|c|c|c|c|c|}
\hline 36 & A37 & A. hirtum (Lam). Sweet & 10-11-2019 & Najran Hadadah & $17^{\circ} 35^{\prime} 44^{\prime \prime} \mathrm{N}$ & $43^{\circ} 44^{\prime} 49^{\prime \prime} \mathrm{E}$ \\
\hline 37 & A38 & A. pannosum (Frost. Fil.) Schltdl & $12-11-2019$ & Adum & $20^{\circ} 27^{\prime} 7^{\prime \prime} \mathrm{N}$ & $40^{\circ} 52^{\prime} 42^{\prime \prime} \mathrm{E}$ \\
\hline 39 & $\mathrm{~A} 40$ & A. pannosum (Frost. Fil.) Schltdl & $12-11-2019$ & Adum & $20^{\circ} 26^{\prime} 7^{\prime \prime} \mathrm{N}$ & $40^{\circ} 52^{\prime} 43^{\prime \prime} \mathrm{E}$ \\
\hline 40 & A41 & A. pannosum (Frost. Fil.) Schltdl & 20-11-2019 & Al Dammam & $26^{\circ} 25^{\prime} 12^{\prime \prime} \mathrm{N}$ & $50^{\circ} 04^{\prime} 44^{\prime \prime} \mathrm{E}$ \\
\hline 41 & D63 & A. pannosum (Frost. Fil.) Schltdl & $3-5-2018$ & Alwasha-Al Taif & $21^{\circ} 14^{\prime} 52^{\prime \prime} \mathrm{N}$ & $40^{\circ} 25^{\prime} 54^{\prime \prime} \mathrm{E}$ \\
\hline 42 & D64 & A. fruticosum Guill \& Perr & $3-5-2018$ & Alwasha-Al Taif & $21^{\circ} 14^{\prime} 52^{\prime \prime} \mathrm{N}$ & $40^{\circ} 25^{\prime} 54^{\prime \prime} \mathrm{E}$ \\
\hline 43 & D74 & A. bidentatum Hochest. Ex A. Rich & $19-10-2018$ & Al Baha Al Aqiq & $20^{\circ} 13^{\prime} 19^{\prime \prime} \mathrm{N}$ & $41^{\circ} 36^{\prime} 20^{\prime \prime} \mathrm{E}$ \\
\hline
\end{tabular}

specimens were identified at the herbarium of the King Abdulaziz University, Jeddah, Saudi Arabia. Voucher specimens were deposited in the herbarium for reference and further studies.

\subsection{Macromorphological Investigation}

36 Morphological characteristics include 26 quantitative characters (Table 2) and 10 qualitative characters (Table 3 ) and (Table 4) such as leaves, flowers and fruits characters, were examined and scored using a light microscope M6C-10 and hand lens X10. The average measurements for ten mature leaves, flowers, and fruits were taken for each sample. Measurements are given in centimetres and millimetres. Also photographs of relevant morphological features were taken.

\subsection{Numerical Analysis}

Based on the qualitative and quantitative characters were analysed the Principle Coordinates Analysis (PCoA) and the Unweighted Pair Group Method with Arithmetic Algorithm (UPGMA) based on the similarity matrix generated using Gower's general similarity coefficient [25]. Both analyses were performed using the package MVSP version 3.1 [26]. While, based on quantitative characters were analysed the Principle Component Analysis (PCA) using Minitab package 15.1.30 [27].

The Cluster Analysis. One of the commonly used approaches is the unweighted pair group method with arithmetic algorithm (UPGMA) [28] approach to cluster analysis and is also a simple method for tree construction. The UPGMA was implemented using the MVSP version 3.1 package [26], and the clustering introduced the general similarity coefficient of Gower [25].

The Principle Coordinate Analysis (PCoA): The Principle Coordinate Analysis was conducted employ version 3.1 of the MVSP package [26], utilize the same matrix of similarity generated using the general coefficient of similarity of Gower [25].

The Principle Component Analysis (PCA): For quantitative morphological characteristics (Table 5), Principle Component Analysis was performed and the 
Table 2. 26 quantitative morphological characters of 43 Abutilon samples.

\begin{tabular}{cccc}
\hline No. & Characters & No. & Characters \\
\hline 1. & Plant high $(\mathrm{m})$ & 14. & Length of stamens $(\mathrm{mm})$ \\
2. & Length of stipulate $(\mathrm{mm})$ & 15. & Length of filaments $(\mathrm{mm})$ \\
3. & Length of petiolate $(\mathrm{mm})$ & 16. & Number of filaments \\
4. & Length of midrib $(\mathrm{mm})$ & 17. & Length of style $(\mathrm{mm})$ \\
5. & Length of leaf $(\mathrm{mm})$ & 18. & Length of mericarp $(\mathrm{mm})$ \\
6. & Length of petal $(\mathrm{mm})$ & 19. & Width of mericarp $(\mathrm{mm})$ \\
7. & Width of petal $(\mathrm{mm})$ & 20. & Number of mericarps \\
8. & Flower diameter $(\mathrm{mm})$ & 21. & Length of fruit $(\mathrm{mm})$ \\
9. & Length of upper part of the pedicle $(\mathrm{mm})$ & 22. & Width of fruit $(\mathrm{mm})$ \\
10. & Length of lower part of the pedicle $(\mathrm{mm})$ & 23. & Length of seed $(\mathrm{mm})$ \\
11. & Length of whole pedicle $(\mathrm{mm})$ & 24. & Width of seed $(\mathrm{mm})$ \\
12. & Length of sepals $(\mathrm{mm})$ & 25. & Number of seeds per mericarp \\
13. & Width of sepals $(\mathrm{mm})$ & 26. & Length of awn on seed $(\mathrm{mm})$ \\
\hline
\end{tabular}

Table 3. Ten qualitative morphological characters and character states used in morphometric analysis of Abutilon samples.

\begin{tabular}{|c|c|c|c|}
\hline No. & Character & Character state & Code \\
\hline \multirow{3}{*}{1} & \multirow{3}{*}{ Habit } & Shrub $\leq 1 \mathrm{~m}$ & 1 \\
\hline & & Shrub $>1 \mathrm{~m} \leq 2.5 \mathrm{~m}$ & 2 \\
\hline & & Shrub $>2.5 \mathrm{~m}$ & 3 \\
\hline \multirow{4}{*}{2} & \multirow{4}{*}{ Leaf surface } & Glabrous & 1 \\
\hline & & Pubescent to tomentose & 2 \\
\hline & & Velvety & 3 \\
\hline & & Hairy & 4 \\
\hline \multirow{3}{*}{3} & \multirow{3}{*}{ Leaf colour } & Green & 1 \\
\hline & & Greyish green & 2 \\
\hline & & Yellowish green & 3 \\
\hline \multirow{2}{*}{4} & \multirow{2}{*}{ Leaf shape } & Cordate & 1 \\
\hline & & Ovate to broadly ovate & 2 \\
\hline \multirow{4}{*}{5} & \multirow{4}{*}{ Leaf apex } & Acute & 1 \\
\hline & & Cuspidate & 2 \\
\hline & & Acute to cuspidate & 3 \\
\hline & & Obtuse & 4 \\
\hline \multirow{3}{*}{6} & \multirow{3}{*}{ Leaf base } & Truncate & 1 \\
\hline & & Cordate & 2 \\
\hline & & Truncate to Cordate & 3 \\
\hline \multirow{3}{*}{7} & \multirow{3}{*}{ Leaf margin } & Double serrate & 1 \\
\hline & & Double Denticulate to Dentate & 2 \\
\hline & & Entire & 3 \\
\hline \multirow{3}{*}{8} & \multirow{3}{*}{ Flower colour } & Yellow & 1 \\
\hline & & Yellow with a red centre & 2 \\
\hline & & Yellow pale & 3 \\
\hline \multirow{2}{*}{9} & \multirow{2}{*}{ Fruit shape } & Globose schizocarp & 1 \\
\hline & & Cylindric schizocarp & 2 \\
\hline \multirow{2}{*}{10} & \multirow{2}{*}{ Mericarp shape } & Reniform with rounded apex & 1 \\
\hline & & Reniform with pointed apex & 2 \\
\hline
\end{tabular}


Table 4. Qualitative morphological characters and character states used in morphometric analysis of Saudi Arabian Abutilon plants.

\begin{tabular}{|c|c|c|c|c|c|c|c|c|c|c|c|}
\hline 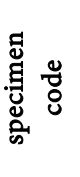 & 泀 & 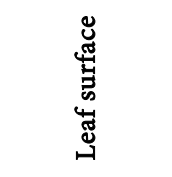 & 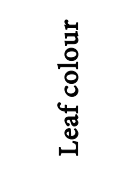 & 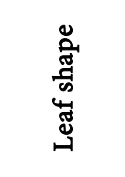 & 壱 & 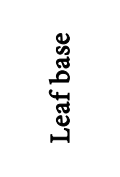 & 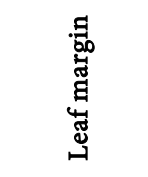 & 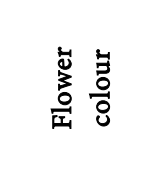 & 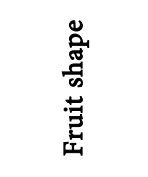 & 营 & 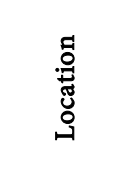 \\
\hline $\mathrm{A} 1$ & $\begin{array}{l}\text { Shrub }>1 \mathrm{~m} \\
\quad \leq 2.5 \mathrm{~m}\end{array}$ & Velvety & Green & $\begin{array}{c}\text { Ovate to } \\
\text { broadly } \\
\text { ovate }\end{array}$ & $\begin{array}{l}\text { Acute to } \\
\text { Cuspidate }\end{array}$ & Cordate & $\begin{array}{c}\text { Double } \\
\text { denticulate } \\
\text { to dentate }\end{array}$ & $\begin{array}{l}\text { Yellow with } \\
\text { red center }\end{array}$ & $\begin{array}{c}\text { Globose } \\
\text { Schizocarp }\end{array}$ & $\begin{array}{l}\text { Reniform with } \\
\text { rounded apex }\end{array}$ & Khulais \\
\hline A2 & $\begin{array}{l}\text { Shrub }>1 \mathrm{~m} \\
\quad \leq 2.5 \mathrm{~m}\end{array}$ & Velvety & Green & Cordate & $\begin{array}{l}\text { Acute to } \\
\text { Cuspidate }\end{array}$ & Cordate & $\begin{array}{c}\text { Double } \\
\text { denticulate } \\
\text { to dentate }\end{array}$ & $\begin{array}{l}\text { Yellow with } \\
\text { red center }\end{array}$ & $\begin{array}{c}\text { Globose } \\
\text { Schizocarp }\end{array}$ & $\begin{array}{l}\text { Reniform with } \\
\text { rounded apex }\end{array}$ & Khulais \\
\hline $\mathrm{A} 3$ & $\begin{array}{l}\text { Shrub }>1 \mathrm{~m} \\
\quad \leq 2.5 \mathrm{~m}\end{array}$ & Velvety & Green & $\begin{array}{c}\text { Ovate to } \\
\text { broadly } \\
\text { ovate }\end{array}$ & $\begin{array}{l}\text { Acute to } \\
\text { Cuspidate }\end{array}$ & Truncate & $\begin{array}{c}\text { Double } \\
\text { denticulate } \\
\text { to dentate }\end{array}$ & $\begin{array}{l}\text { Yellow with } \\
\text { red center }\end{array}$ & $\begin{array}{l}\text { Globose } \\
\text { Schizocarp }\end{array}$ & $\begin{array}{l}\text { Reniform with } \\
\text { rounded apex }\end{array}$ & Khulais \\
\hline A4 & $\begin{array}{l}\text { Shrub }>1 \mathrm{~m} \\
\quad \leq 2.5 \mathrm{~m}\end{array}$ & $\begin{array}{c}\text { Pubescent to } \\
\text { tomentosa }\end{array}$ & $\begin{array}{l}\text { Yellowish } \\
\text { green }\end{array}$ & $\begin{array}{c}\text { Ovate to } \\
\text { broadly } \\
\text { ovate }\end{array}$ & Cuspidate & Cordate & $\begin{array}{l}\text { Double } \\
\text { serrate }\end{array}$ & $\begin{array}{l}\text { Yellow with } \\
\text { red center }\end{array}$ & $\begin{array}{l}\text { Globose } \\
\text { Schizocarp }\end{array}$ & $\begin{array}{l}\text { Reniform with } \\
\text { rounded apex }\end{array}$ & Madinah \\
\hline A5 & Shrub $\leq 1 \mathrm{~m}$ & $\begin{array}{c}\text { Pubescent to } \\
\text { tomentosa }\end{array}$ & $\begin{array}{l}\text { Yellowish } \\
\text { green }\end{array}$ & $\begin{array}{c}\text { Ovate to } \\
\text { broadly } \\
\text { ovate }\end{array}$ & $\begin{array}{l}\text { Acute to } \\
\text { Cuspidate }\end{array}$ & Cordate & $\begin{array}{c}\text { Double } \\
\text { denticulate } \\
\text { to dentate }\end{array}$ & $\begin{array}{l}\text { Yellow with } \\
\text { red center }\end{array}$ & $\begin{array}{c}\text { Globose } \\
\text { Schizocarp }\end{array}$ & $\begin{array}{l}\text { Reniform with } \\
\text { rounded apex }\end{array}$ & Madinah \\
\hline A6 & $\begin{array}{l}\text { Shrub }>1 \mathrm{~m} \\
\quad \leq 2.5 \mathrm{~m}\end{array}$ & $\begin{array}{c}\text { Pubescent to } \\
\text { tomentosa }\end{array}$ & $\begin{array}{l}\text { Yellowish } \\
\text { green }\end{array}$ & $\begin{array}{c}\text { Ovate to } \\
\text { broadly } \\
\text { ovate }\end{array}$ & $\begin{array}{l}\text { Acute to } \\
\text { Cuspidate }\end{array}$ & Cordate & $\begin{array}{c}\text { Double } \\
\text { denticulate } \\
\text { to dentate }\end{array}$ & $\begin{array}{l}\text { Yellow with } \\
\text { red center }\end{array}$ & $\begin{array}{l}\text { Globose } \\
\text { Schizocarp }\end{array}$ & $\begin{array}{l}\text { Reniform with } \\
\text { rounded apex }\end{array}$ & Madinah \\
\hline A7 & $\begin{array}{l}\text { Shrub }>1 \mathrm{~m} \\
\quad \leq 2.5 \mathrm{~m}\end{array}$ & $\begin{array}{l}\text { Pubescent to } \\
\text { tomentosa }\end{array}$ & $\begin{array}{l}\text { Yellowish } \\
\text { green }\end{array}$ & $\begin{array}{c}\text { Ovate to } \\
\text { broadly } \\
\text { ovate }\end{array}$ & $\begin{array}{l}\text { Acute to } \\
\text { Cuspidate }\end{array}$ & Cordate & $\begin{array}{c}\text { Double } \\
\text { denticulate } \\
\text { to dentate }\end{array}$ & $\begin{array}{l}\text { Yellow with } \\
\text { red center }\end{array}$ & $\begin{array}{l}\text { Globose } \\
\text { Schizocarp }\end{array}$ & $\begin{array}{l}\text { Reniform with } \\
\text { rounded apex }\end{array}$ & Jeddah \\
\hline A8 & $\begin{array}{l}\text { Shrub }>1 \mathrm{~m} \\
\quad \leq 2.5 \mathrm{~m}\end{array}$ & $\begin{array}{l}\text { Pubescent to } \\
\text { tomentosa }\end{array}$ & Green & $\begin{array}{c}\text { Ovate to } \\
\text { broadly } \\
\text { ovate }\end{array}$ & $\begin{array}{l}\text { Acute to } \\
\text { Cuspidate }\end{array}$ & Cordate & $\begin{array}{c}\text { Double } \\
\text { denticulate } \\
\text { to dentate }\end{array}$ & $\begin{array}{l}\text { Yellow with } \\
\text { red center }\end{array}$ & $\begin{array}{c}\text { Globose } \\
\text { Schizocarp }\end{array}$ & $\begin{array}{c}\text { Reniform with } \\
\text { rounded apex }\end{array}$ & Jeddah \\
\hline A9 & $\begin{array}{l}\text { Shrub }>1 \mathrm{~m} \\
\quad \leq 2.5 \mathrm{~m}\end{array}$ & $\begin{array}{c}\text { Pubescent to } \\
\text { tomentosa }\end{array}$ & $\begin{array}{l}\text { Yellowish } \\
\text { green }\end{array}$ & $\begin{array}{c}\text { Ovate to } \\
\text { broadly } \\
\text { ovate }\end{array}$ & Cuspidate & Cordate & $\begin{array}{l}\text { Double } \\
\text { serrate }\end{array}$ & $\begin{array}{l}\text { Yellow with } \\
\text { red center }\end{array}$ & $\begin{array}{l}\text { Globose } \\
\text { Schizocarp }\end{array}$ & $\begin{array}{l}\text { Reniform with } \\
\text { rounded apex }\end{array}$ & Jeddah \\
\hline $\mathrm{A} 10$ & $\begin{array}{l}\text { Shrub }>1 \mathrm{~m} \\
\quad \leq 2.5 \mathrm{~m}\end{array}$ & $\begin{array}{c}\text { Pubescent to } \\
\text { tomentosa }\end{array}$ & Green & Cordate & Acute & Cordate & $\begin{array}{c}\text { Double } \\
\text { denticulate } \\
\text { to dentate }\end{array}$ & $\begin{array}{l}\text { Yellow with } \\
\text { red center }\end{array}$ & $\begin{array}{l}\text { Globose } \\
\text { Schizocarp }\end{array}$ & $\begin{array}{l}\text { Reniform with } \\
\text { rounded apex }\end{array}$ & Jeddah \\
\hline A11 & $\begin{array}{l}\text { Shrub }>1 \mathrm{~m} \\
\quad \leq 2.5 \mathrm{~m}\end{array}$ & $\begin{array}{c}\text { Pubescent to } \\
\text { tomentosa }\end{array}$ & Green & $\begin{array}{c}\text { Ovate to } \\
\text { broadly } \\
\text { ovate }\end{array}$ & $\begin{array}{l}\text { Acute to } \\
\text { Cuspidate }\end{array}$ & Cordate & $\begin{array}{l}\text { Double } \\
\text { serrate }\end{array}$ & $\begin{array}{l}\text { Yellow with } \\
\text { red center }\end{array}$ & $\begin{array}{l}\text { Globose } \\
\text { Schizocarp }\end{array}$ & $\begin{array}{l}\text { Reniform with } \\
\text { rounded apex }\end{array}$ & Jeddah \\
\hline $\mathrm{A} 12$ & $\begin{array}{l}\text { Shrub }>1 \mathrm{~m} \\
\quad \leq 2.5 \mathrm{~m}\end{array}$ & $\begin{array}{l}\text { Pubescent to } \\
\text { tomentosa }\end{array}$ & Green & $\begin{array}{c}\text { Ovate to } \\
\text { broadly } \\
\text { ovate }\end{array}$ & Acute & Cordate & $\begin{array}{c}\text { Double } \\
\text { denticulate } \\
\text { to dentate }\end{array}$ & $\begin{array}{l}\text { Yellow with } \\
\text { red center }\end{array}$ & $\begin{array}{l}\text { Globose } \\
\text { Schizocarp }\end{array}$ & $\begin{array}{l}\text { Reniform with } \\
\text { rounded apex }\end{array}$ & Al baha \\
\hline A13 & $\begin{array}{l}\text { Shrub }>1 \mathrm{~m} \\
\quad \leq 2.5 \mathrm{~m}\end{array}$ & $\begin{array}{l}\text { Pubescent to } \\
\text { tomentosa }\end{array}$ & Green & Cordate & Acute & Cordate & $\begin{array}{c}\text { Double } \\
\text { denticulate } \\
\text { to dentate }\end{array}$ & $\begin{array}{l}\text { Yellow with } \\
\text { red center }\end{array}$ & $\begin{array}{l}\text { Globose } \\
\text { Schizocarp }\end{array}$ & $\begin{array}{l}\text { Reniform with } \\
\text { rounded apex }\end{array}$ & Al baha \\
\hline A14 & Shrub $\leq 1 \mathrm{~m}$ & $\begin{array}{l}\text { Pubescent to } \\
\text { tomentosa }\end{array}$ & Green & Cordate & Obtuse & Cordate & $\begin{array}{c}\text { Double } \\
\text { denticulate } \\
\text { to dentate }\end{array}$ & $\begin{array}{l}\text { Yellow with } \\
\text { red center }\end{array}$ & $\begin{array}{l}\text { Globose } \\
\text { Schizocarp }\end{array}$ & $\begin{array}{l}\text { Reniform with } \\
\text { rounded apex }\end{array}$ & Al baha \\
\hline A15 & $\begin{array}{l}\text { Shrub }>1 \mathrm{~m} \\
\quad \leq 2.5 \mathrm{~m}\end{array}$ & $\begin{array}{c}\text { Pubescent to } \\
\text { tomentosa }\end{array}$ & Green & Cordate & Obtuse & Cordate & $\begin{array}{c}\text { Double } \\
\text { denticulate } \\
\text { to dentate }\end{array}$ & $\begin{array}{l}\text { Yellow with } \\
\text { red center }\end{array}$ & $\begin{array}{l}\text { Globose } \\
\text { Schizocarp }\end{array}$ & $\begin{array}{l}\text { Reniform with } \\
\text { rounded apex }\end{array}$ & Al baha \\
\hline
\end{tabular}




\section{Continued}

\begin{tabular}{|c|c|c|c|c|c|c|c|c|c|c|c|}
\hline A16 & $\begin{array}{l}\text { Shrub }>1 \mathrm{~m} \\
\quad \leq 2.5 \mathrm{~m}\end{array}$ & $\begin{array}{c}\text { Pubescent to } \\
\text { tomentosa }\end{array}$ & Green & $\begin{array}{c}\text { Ovate to } \\
\text { broadly } \\
\text { ovate }\end{array}$ & Acute & Cordate & $\begin{array}{l}\text { Double } \\
\text { serrate }\end{array}$ & $\begin{array}{l}\text { Yellow with } \\
\text { red center }\end{array}$ & $\begin{array}{c}\text { Globose } \\
\text { Schizocarp }\end{array}$ & $\begin{array}{l}\text { Reniform with } \\
\text { rounded apex }\end{array}$ & Al baha \\
\hline A17 & $\begin{array}{l}\text { Shrub }>1 \mathrm{~m} \\
\quad \leq 2.5 \mathrm{~m}\end{array}$ & $\begin{array}{c}\text { Pubescent to } \\
\text { tomentosa }\end{array}$ & Green & Cordate & $\begin{array}{l}\text { Acute to } \\
\text { Cuspidate }\end{array}$ & Cordate & $\begin{array}{c}\text { Double } \\
\text { denticulate } \\
\text { to dentate }\end{array}$ & $\begin{array}{l}\text { Yellow with } \\
\text { red center }\end{array}$ & $\begin{array}{c}\text { Globose } \\
\text { Schizocarp }\end{array}$ & $\begin{array}{l}\text { Reniform with } \\
\text { rounded apex }\end{array}$ & Jeddah \\
\hline A18 & $\begin{array}{l}\text { Shrub }>1 \mathrm{~m} \\
\quad \leq 2.5 \mathrm{~m}\end{array}$ & $\begin{array}{c}\text { Pubescent to } \\
\text { tomentosa }\end{array}$ & Green & $\begin{array}{c}\text { Ovate to } \\
\text { broadly } \\
\text { ovate }\end{array}$ & Acute & $\begin{array}{l}\text { Cordate to } \\
\text { truncate }\end{array}$ & $\begin{array}{c}\text { Double } \\
\text { denticulate } \\
\text { to dentate }\end{array}$ & Yellow & $\begin{array}{c}\text { Globose } \\
\text { Schizocarp }\end{array}$ & $\begin{array}{l}\text { Reniform with } \\
\text { rounded apex }\end{array}$ & Jeddah \\
\hline A19 & $\begin{array}{c}\text { Shrub }>2.5 \\
\mathrm{~m}\end{array}$ & $\begin{array}{c}\text { Pubescent to } \\
\text { tomentosa }\end{array}$ & Green & $\begin{array}{c}\text { Ovate to } \\
\text { broadly } \\
\text { ovate }\end{array}$ & Obtuse & Cordate & $\begin{array}{c}\text { Double } \\
\text { denticulate } \\
\text { to dentate }\end{array}$ & $\begin{array}{l}\text { Yellow with } \\
\text { red center }\end{array}$ & $\begin{array}{c}\text { Globose } \\
\text { Schizocarp }\end{array}$ & $\begin{array}{l}\text { Reniform with } \\
\text { rounded apex }\end{array}$ & Jeddah \\
\hline A20 & $\begin{array}{l}\text { Shrub }>1 \mathrm{~m} \\
\quad \leq 2.5 \mathrm{~m}\end{array}$ & $\begin{array}{c}\text { Pubescent to } \\
\text { tomentosa }\end{array}$ & Green & $\begin{array}{c}\text { Ovate to } \\
\text { broadly } \\
\text { ovate }\end{array}$ & $\begin{array}{l}\text { Acute to } \\
\text { Cuspidate }\end{array}$ & Cordate & $\begin{array}{c}\text { Double } \\
\text { denticulate } \\
\text { to dentate }\end{array}$ & $\begin{array}{l}\text { Yellow with } \\
\text { red center }\end{array}$ & $\begin{array}{c}\text { Globose } \\
\text { Schizocarp }\end{array}$ & $\begin{array}{l}\text { Reniform with } \\
\text { rounded apex }\end{array}$ & Makka \\
\hline A21 & $\begin{array}{l}\text { Shrub }>1 \mathrm{~m} \\
\quad \leq 2.5 \mathrm{~m}\end{array}$ & $\begin{array}{c}\text { Pubescent to } \\
\text { tomentosa }\end{array}$ & Green & $\begin{array}{c}\text { Ovate to } \\
\text { broadly } \\
\text { ovate }\end{array}$ & $\begin{array}{l}\text { Acute to } \\
\text { Cuspidate }\end{array}$ & Cordate & $\begin{array}{c}\text { Double } \\
\text { denticulate } \\
\text { to dentate }\end{array}$ & $\begin{array}{l}\text { Yellow with } \\
\text { red center }\end{array}$ & $\begin{array}{l}\text { Globose } \\
\text { Schizocarp }\end{array}$ & $\begin{array}{l}\text { Reniform with } \\
\text { rounded apex }\end{array}$ & Makka \\
\hline A22 & $\begin{array}{l}\text { Shrub }>1 \mathrm{~m} \\
\quad \leq 2.5 \mathrm{~m}\end{array}$ & $\begin{array}{c}\text { Pubescent to } \\
\text { tomentosa }\end{array}$ & Green & $\begin{array}{c}\text { Ovate to } \\
\text { broadly } \\
\text { ovate }\end{array}$ & $\begin{array}{l}\text { Acute to } \\
\text { Cuspidate }\end{array}$ & Cordate & $\begin{array}{c}\text { Double } \\
\text { denticulate } \\
\text { to dentate }\end{array}$ & $\begin{array}{l}\text { Yellow with } \\
\text { red center }\end{array}$ & $\begin{array}{c}\text { Globose } \\
\text { Schizocarp }\end{array}$ & $\begin{array}{l}\text { Reniform with } \\
\text { rounded apex }\end{array}$ & Makka \\
\hline A23 & $\begin{array}{l}\text { Shrub }>1 \mathrm{~m} \\
\quad \leq 2.5 \mathrm{~m}\end{array}$ & $\begin{array}{l}\text { Pubescent to } \\
\text { tomentosa }\end{array}$ & Green & $\begin{array}{c}\text { Ovate to } \\
\text { broadly } \\
\text { ovate }\end{array}$ & Acute & $\begin{array}{l}\text { Cordate to } \\
\text { truncate }\end{array}$ & $\begin{array}{c}\text { Double } \\
\text { denticulate } \\
\text { to dentate }\end{array}$ & $\begin{array}{l}\text { Yellow with } \\
\text { red center }\end{array}$ & $\begin{array}{l}\text { Globose } \\
\text { Schizocarp }\end{array}$ & $\begin{array}{l}\text { Reniform with } \\
\text { rounded apex }\end{array}$ & Makka \\
\hline A 24 & $\begin{array}{l}\text { Shrub }>1 \mathrm{~m} \\
\quad \leq 2.5 \mathrm{~m}\end{array}$ & $\begin{array}{c}\text { Pubescent to } \\
\text { tomentosa }\end{array}$ & $\begin{array}{l}\text { Yellowish } \\
\text { green }\end{array}$ & Cordate & Acute & Cordate & $\begin{array}{c}\text { Double } \\
\text { denticulate } \\
\text { to dentate }\end{array}$ & $\begin{array}{l}\text { Yellow with } \\
\text { red center }\end{array}$ & $\begin{array}{c}\text { Globose } \\
\text { Schizocarp }\end{array}$ & $\begin{array}{l}\text { Reniform with } \\
\text { rounded apex }\end{array}$ & Makka \\
\hline A25 & Shrub $\leq 1 \mathrm{~m}$ & $\begin{array}{c}\text { Pubescent to } \\
\text { tomentosa }\end{array}$ & $\begin{array}{l}\text { Yellowish } \\
\text { green }\end{array}$ & $\begin{array}{c}\text { Ovate to } \\
\text { broadly } \\
\text { ovate }\end{array}$ & $\begin{array}{l}\text { Acute to } \\
\text { Cuspidate }\end{array}$ & Cordate & $\begin{array}{c}\text { Double } \\
\text { denticulate } \\
\text { to dentate }\end{array}$ & $\begin{array}{l}\text { Yellow with } \\
\text { red center }\end{array}$ & $\begin{array}{c}\text { Globose } \\
\text { Schizocarp }\end{array}$ & $\begin{array}{l}\text { Reniform with } \\
\text { rounded apex }\end{array}$ & Makka \\
\hline A26 & $\begin{array}{l}\text { Shrub }>1 \mathrm{~m} \\
\quad \leq 2.5 \mathrm{~m}\end{array}$ & $\begin{array}{l}\text { Pubescent to } \\
\text { tomentosa }\end{array}$ & $\begin{array}{l}\text { Yellowish } \\
\text { green }\end{array}$ & $\begin{array}{c}\text { Ovate to } \\
\text { broadly } \\
\text { ovate }\end{array}$ & Acute & Truncate & $\begin{array}{c}\text { Double } \\
\text { denticulate } \\
\text { to dentate }\end{array}$ & $\begin{array}{l}\text { Yellow with } \\
\text { red center }\end{array}$ & $\begin{array}{c}\text { Globose } \\
\text { Schizocarp }\end{array}$ & $\begin{array}{l}\text { Reniform with } \\
\text { rounded apex }\end{array}$ & Abaha \\
\hline A 27 & $\begin{array}{l}\text { Shrub }>1 \mathrm{~m} \\
\quad \leq 2.5 \mathrm{~m}\end{array}$ & $\begin{array}{l}\text { Pubescent to } \\
\text { tomentosa }\end{array}$ & $\begin{array}{c}\text { Yellowish } \\
\text { green }\end{array}$ & $\begin{array}{c}\text { Ovate to } \\
\text { broadly } \\
\text { ovate }\end{array}$ & $\begin{array}{l}\text { Acute to } \\
\text { Cuspidate }\end{array}$ & Cordate & $\begin{array}{c}\text { Double } \\
\text { denticulate } \\
\text { to dentate }\end{array}$ & Yellow & $\begin{array}{l}\text { Globose } \\
\text { Schizocarp }\end{array}$ & $\begin{array}{l}\text { Reniform with } \\
\text { rounded apex }\end{array}$ & Abaha \\
\hline A28 & $\begin{array}{l}\text { Shrub }>1 \mathrm{~m} \\
\quad \leq 2.5 \mathrm{~m}\end{array}$ & $\begin{array}{l}\text { Pubescent to } \\
\text { tomentosa }\end{array}$ & $\begin{array}{l}\text { Yellowish } \\
\text { green }\end{array}$ & $\begin{array}{c}\text { Ovate to } \\
\text { broadly } \\
\text { ovate }\end{array}$ & Cuspidate & Cordate & $\begin{array}{c}\text { Double } \\
\text { denticulate } \\
\text { to dentate }\end{array}$ & $\begin{array}{l}\text { Yellow with } \\
\text { red center }\end{array}$ & $\begin{array}{l}\text { Globose } \\
\text { Schizocarp }\end{array}$ & $\begin{array}{l}\text { Reniform with } \\
\text { rounded apex }\end{array}$ & Abaha \\
\hline A29 & Shrub $\leq 1 \mathrm{~m}$ & Hairy & Green & Cordate & Cuspidate & Cordate & $\begin{array}{c}\text { Double } \\
\text { denticulate } \\
\text { to dentate }\end{array}$ & Yellow & $\begin{array}{l}\text { Cylindric } \\
\text { Schizocarp }\end{array}$ & $\begin{array}{l}\text { Reniform with } \\
\text { pointed apex }\end{array}$ & Abaha \\
\hline A30 & Shrub $\leq 1 \mathrm{~m}$ & Hairy & Green & $\begin{array}{c}\text { Ovate to } \\
\text { broadly } \\
\text { ovate }\end{array}$ & Cuspidate & Cordate & $\begin{array}{c}\text { Double } \\
\text { denticulate } \\
\text { to dentate }\end{array}$ & Yellow & $\begin{array}{l}\text { Cylindric } \\
\text { Schizocarp }\end{array}$ & $\begin{array}{l}\text { Reniform with } \\
\text { pointed apex }\end{array}$ & Abaha \\
\hline A 31 & $\begin{array}{l}\text { Shrub }>1 \mathrm{~m} \\
\quad \leq 2.5 \mathrm{~m}\end{array}$ & Hairy & Green & $\begin{array}{c}\text { Ovate to } \\
\text { broadly } \\
\text { ovate }\end{array}$ & Cuspidate & Cordate & $\begin{array}{c}\text { Double } \\
\text { denticulate } \\
\text { to dentate }\end{array}$ & Yellow & $\begin{array}{l}\text { Cylindric } \\
\text { Schizocarp }\end{array}$ & $\begin{array}{l}\text { Reniform with } \\
\text { pointed apex }\end{array}$ & Abaha \\
\hline
\end{tabular}




\section{Continued}

\begin{tabular}{|c|c|c|c|c|c|c|c|c|c|c|c|}
\hline A 32 & $\begin{array}{c}\text { Shrub }>2.5 \\
\mathrm{~m}\end{array}$ & $\begin{array}{l}\text { Pubescent to } \\
\text { tomentosa }\end{array}$ & Green & $\begin{array}{l}\text { Ovate to } \\
\text { broadly } \\
\text { ovate }\end{array}$ & Acute & $\begin{array}{l}\text { Cordate to } \\
\text { truncate }\end{array}$ & $\begin{array}{c}\text { Double } \\
\text { denticulate } \\
\text { to dentate }\end{array}$ & $\begin{array}{l}\text { Yellow with } \\
\text { red center }\end{array}$ & $\begin{array}{c}\text { Globose } \\
\text { Schizocarp }\end{array}$ & $\begin{array}{l}\text { Reniform with } \\
\text { rounded apex }\end{array}$ & Abaha \\
\hline A33 & $\begin{array}{c}\text { Shrub }>2.5 \\
\mathrm{~m}\end{array}$ & $\begin{array}{l}\text { Pubescent to } \\
\text { tomentosa }\end{array}$ & Green & $\begin{array}{l}\text { Ovate to } \\
\text { broadly } \\
\text { ovate }\end{array}$ & Cuspidate & $\begin{array}{l}\text { Cordate to } \\
\text { truncate }\end{array}$ & $\begin{array}{c}\text { Double } \\
\text { denticulate } \\
\text { to dentate }\end{array}$ & Yellow & $\begin{array}{c}\text { Globose } \\
\text { Schizocarp }\end{array}$ & $\begin{array}{l}\text { Reniform with } \\
\text { rounded apex }\end{array}$ & Abaha \\
\hline A35 & $\begin{array}{c}\text { Shrub }>1 \mathrm{~m} \\
\leq 2.5 \mathrm{~m}\end{array}$ & $\begin{array}{l}\text { Pubescent to } \\
\text { tomentosa }\end{array}$ & $\begin{array}{l}\text { Yellowish } \\
\text { green }\end{array}$ & Cordate & $\begin{array}{l}\text { Acute to } \\
\text { Cuspidate }\end{array}$ & $\begin{array}{l}\text { Cordate to } \\
\text { truncate }\end{array}$ & $\begin{array}{c}\text { Double } \\
\text { denticulate } \\
\text { to dentate }\end{array}$ & $\begin{array}{l}\text { Yellow with } \\
\text { red center }\end{array}$ & $\begin{array}{c}\text { Globose } \\
\text { Schizocarp }\end{array}$ & $\begin{array}{l}\text { Reniform with } \\
\text { rounded apex }\end{array}$ & $\begin{array}{c}\text { Al } \\
\text { Qunfudhah }\end{array}$ \\
\hline A36 & $\begin{array}{c}\text { Shrub }>2.5 \\
\mathrm{~m}\end{array}$ & Velvety & Green & $\begin{array}{l}\text { Ovate to } \\
\text { broadly } \\
\text { ovate }\end{array}$ & $\begin{array}{l}\text { Acute to } \\
\text { Cuspidate }\end{array}$ & $\begin{array}{l}\text { Cordate to } \\
\text { truncate }\end{array}$ & $\begin{array}{c}\text { Double } \\
\text { denticulate } \\
\text { to dentate }\end{array}$ & $\begin{array}{l}\text { Yellow with } \\
\text { red center }\end{array}$ & $\begin{array}{c}\text { Globose } \\
\text { Schizocarp }\end{array}$ & $\begin{array}{l}\text { Reniform with } \\
\text { rounded apex }\end{array}$ & $\begin{array}{c}\text { Al } \\
\text { Qunfudhah }\end{array}$ \\
\hline A 37 & $\begin{array}{c}\text { Shrub }>1 \mathrm{~m} \\
\leq 2.5 \mathrm{~m}\end{array}$ & Velvety & Green & $\begin{array}{l}\text { Ovate to } \\
\text { broadly } \\
\text { ovate }\end{array}$ & $\begin{array}{l}\text { Acute to } \\
\text { Cuspidate }\end{array}$ & Cordate & $\begin{array}{c}\text { Double } \\
\text { denticulate } \\
\text { to dentate }\end{array}$ & Yellow pale & $\begin{array}{l}\text { Cylindric } \\
\text { Schizocarp }\end{array}$ & $\begin{array}{l}\text { Reniform with } \\
\text { pointed apex }\end{array}$ & Najran \\
\hline A38 & $\begin{array}{c}\text { Shrub }>1 \mathrm{~m} \\
\leq 2.5 \mathrm{~m}\end{array}$ & $\begin{array}{l}\text { Pubescent to } \\
\text { tomentosa }\end{array}$ & Green & Cordate & Cuspidate & Cordate & $\begin{array}{c}\text { Double } \\
\text { denticulate } \\
\text { to dentate }\end{array}$ & $\begin{array}{l}\text { Yellow with } \\
\text { red center }\end{array}$ & $\begin{array}{c}\text { Globose } \\
\text { Schizocarp }\end{array}$ & $\begin{array}{l}\text { Reniform with } \\
\text { rounded apex }\end{array}$ & Adum \\
\hline A39 & $\begin{array}{c}\text { Shrub }>1 \mathrm{~m} \\
\leq 2.5 \mathrm{~m}\end{array}$ & $\begin{array}{l}\text { Pubescent to } \\
\text { tomentosa }\end{array}$ & Green & Cordate & $\begin{array}{l}\text { Acute to } \\
\text { Cuspidate }\end{array}$ & Cordate & $\begin{array}{c}\text { Double } \\
\text { denticulate } \\
\text { to dentate }\end{array}$ & $\begin{array}{l}\text { Yellow with } \\
\text { red center }\end{array}$ & $\begin{array}{c}\text { Globose } \\
\text { Schizocarp }\end{array}$ & $\begin{array}{l}\text { Reniform with } \\
\text { rounded apex }\end{array}$ & Adum \\
\hline $\mathrm{A} 40$ & $\begin{array}{c}\text { Shrub }>1 \mathrm{~m} \\
\leq 2.5 \mathrm{~m}\end{array}$ & $\begin{array}{l}\text { Pubescent to } \\
\text { tomentosa }\end{array}$ & Green & $\begin{array}{l}\text { Ovate to } \\
\text { broadly } \\
\text { ovate }\end{array}$ & Cuspidate & Cordate & $\begin{array}{c}\text { Double } \\
\text { denticulate } \\
\text { to dentate }\end{array}$ & $\begin{array}{l}\text { Yellow with } \\
\text { red center }\end{array}$ & - & - & Adum \\
\hline A 41 & $\begin{array}{c}\text { Shrub }>1 \mathrm{~m} \\
\leq 2.5 \mathrm{~m}\end{array}$ & Velvety & Green & $\begin{array}{l}\text { Ovate to } \\
\text { broadly } \\
\text { ovate }\end{array}$ & Cuspidate & $\begin{array}{l}\text { Cordate to } \\
\text { truncate }\end{array}$ & $\begin{array}{c}\text { Double } \\
\text { denticulate } \\
\text { to dentate }\end{array}$ & $\begin{array}{l}\text { Yellow with } \\
\text { red center }\end{array}$ & $\begin{array}{c}\text { Globose } \\
\text { Schizocarp }\end{array}$ & $\begin{array}{l}\text { Reniform with } \\
\text { rounded apex }\end{array}$ & $\begin{array}{l}\text { Al Dam- } \\
\text { mam }\end{array}$ \\
\hline D63 & - & $\begin{array}{l}\text { Pubescent to } \\
\text { tomentosa }\end{array}$ & Green & $\begin{array}{l}\text { Ovate to } \\
\text { broadly } \\
\text { ovate }\end{array}$ & Cuspidate & Cordate & $\begin{array}{c}\text { Double } \\
\text { denticulate } \\
\text { to dentate }\end{array}$ & $\begin{array}{l}\text { Yellow with } \\
\text { red center }\end{array}$ & $\begin{array}{c}\text { Globose } \\
\text { Schizocarp }\end{array}$ & $\begin{array}{l}\text { Reniform with } \\
\text { rounded apex }\end{array}$ & Al Taif \\
\hline D64 & - & Glabrous & $\begin{array}{l}\text { Grayish } \\
\text { green }\end{array}$ & $\begin{array}{l}\text { Ovate to } \\
\text { broadly } \\
\text { ovate }\end{array}$ & $\begin{array}{l}\text { Acute to } \\
\text { Cuspidate }\end{array}$ & Cordate & Entire & Yellow pale & $\begin{array}{l}\text { Cylindric } \\
\text { Schizocarp }\end{array}$ & $\begin{array}{l}\text { Reniform with } \\
\text { pointed apex }\end{array}$ & Al Taif \\
\hline D74 & $\begin{array}{c}\text { Shrub }>1 \mathrm{~m} \\
\leq 2.5 \mathrm{~m}\end{array}$ & $\begin{array}{l}\text { Pubescent to } \\
\text { tomentosa }\end{array}$ & $\begin{array}{l}\text { Yellowish } \\
\text { green }\end{array}$ & $\begin{array}{l}\text { Ovate to } \\
\text { broadly } \\
\text { ovate }\end{array}$ & Cuspidate & Cordate & $\begin{array}{c}\text { Double } \\
\text { denticulate } \\
\text { to dentate }\end{array}$ & Yellow & $\begin{array}{l}\text { Cylindric } \\
\text { Schizocarp }\end{array}$ & $\begin{array}{l}\text { Reniform with } \\
\text { pointed apex }\end{array}$ & Al baha \\
\hline
\end{tabular}

Table 5. Qualitative morphological characters and their cods used in morphometric analysis of Saudi Arabian Abutilon plants.

\begin{tabular}{cccccccccccc}
\hline $\begin{array}{c}\text { Sample } \\
\text { No. }\end{array}$ & Specimens No. & Habit & $\begin{array}{c}\text { Leaf } \\
\text { surface }\end{array}$ & Leaf colour Leaf shape & Leaf apex & Leaf base & $\begin{array}{c}\text { Leaf } \\
\text { margin }\end{array}$ & $\begin{array}{c}\text { Flower } \\
\text { colour }\end{array}$ & $\begin{array}{c}\text { Fruit shape } \\
\text { Mericarp } \\
\text { shape }\end{array}$ \\
\hline 1 & A 1 & 2 & 3 & 1 & 2 & 3 & 2 & 2 & 2 & 1 & 1 \\
2 & A 2 & 2 & 3 & 1 & 1 & 3 & 2 & 2 & 2 & 1 & 1 \\
3 & A 3 & 2 & 3 & 1 & 2 & 3 & 1 & 2 & 2 & 1 & 1 \\
4 & A 4 & 2 & 2 & 3 & 2 & 2 & 2 & 1 & 2 & 1 & 1 \\
5 & A 5 & 1 & 2 & 3 & 2 & 3 & 2 & 2 & 2 & 1 & 1 \\
6 & A 6 & 2 & 2 & 3 & 2 & 3 & 2 & 2 & 2 & 1 & 1 \\
\hline
\end{tabular}




\section{Continued}

\begin{tabular}{|c|c|c|c|c|c|c|c|c|c|c|c|}
\hline 7 & A 7 & 2 & 2 & 3 & 2 & 3 & 2 & 2 & 2 & 1 & 1 \\
\hline 8 & A 8 & 2 & 2 & 1 & 2 & 3 & 2 & 2 & 2 & 1 & 1 \\
\hline 9 & A 9 & 2 & 2 & 3 & 2 & 2 & 2 & 1 & 2 & 1 & 1 \\
\hline 10 & A 10 & 2 & 2 & 1 & 1 & 1 & 2 & 2 & 2 & 1 & 1 \\
\hline 11 & A 11 & 2 & 2 & 1 & 2 & 3 & 2 & 1 & 2 & 1 & 1 \\
\hline 12 & A 12 & 2 & 2 & 1 & 2 & 1 & 2 & 2 & 2 & 1 & 1 \\
\hline 13 & A 13 & 2 & 2 & 1 & 1 & 1 & 2 & 2 & 2 & 1 & 1 \\
\hline 14 & A 14 & 1 & 2 & 1 & 1 & 4 & 2 & 2 & 2 & 1 & 1 \\
\hline 15 & A 15 & 2 & 2 & 1 & 1 & 4 & 2 & 2 & 2 & 1 & 1 \\
\hline 16 & A 16 & 2 & 2 & 1 & 2 & 1 & 2 & 1 & 2 & 1 & 1 \\
\hline 17 & A 17 & 2 & 2 & 1 & 1 & 3 & 2 & 2 & 2 & 1 & 1 \\
\hline 18 & A 18 & 2 & 2 & 1 & 2 & 1 & 3 & 2 & 1 & 1 & 1 \\
\hline 19 & A 19 & 3 & 2 & 1 & 2 & 4 & 2 & 2 & 2 & 1 & 1 \\
\hline 20 & A 20 & 2 & 2 & 1 & 2 & 3 & 2 & 2 & 2 & 1 & 1 \\
\hline 21 & A 21 & 2 & 2 & 1 & 2 & 3 & 2 & 2 & 2 & 1 & 1 \\
\hline 22 & A 22 & 2 & 2 & 1 & 2 & 3 & 2 & 2 & 2 & 1 & 1 \\
\hline 23 & A 23 & 2 & 2 & 1 & 2 & 1 & 3 & 2 & 2 & 1 & 1 \\
\hline 24 & A 24 & 2 & 2 & 3 & 1 & 1 & 2 & 2 & 2 & 1 & 1 \\
\hline 25 & A 25 & 1 & 2 & 3 & 2 & 3 & 2 & 2 & 2 & 1 & 1 \\
\hline 26 & A 26 & 2 & 2 & 3 & 2 & 1 & 1 & 2 & 2 & 1 & 1 \\
\hline 27 & A 27 & 2 & 2 & 3 & 2 & 3 & 2 & 2 & 1 & 1 & 1 \\
\hline 28 & A 28 & 2 & 2 & 3 & 2 & 2 & 2 & 2 & 2 & 1 & 1 \\
\hline 29 & А 29 & 1 & 4 & 1 & 1 & 2 & 2 & 2 & 1 & 2 & 2 \\
\hline 30 & A 30 & 1 & 4 & 1 & 2 & 2 & 2 & 2 & 1 & 2 & 2 \\
\hline 31 & A 31 & 2 & 4 & 1 & 2 & 2 & 2 & 2 & 1 & 2 & 2 \\
\hline 32 & A 32 & 3 & 2 & 1 & 2 & 1 & 3 & 2 & 2 & 1 & 1 \\
\hline 33 & A 33 & 3 & 2 & 1 & 2 & 2 & 3 & 2 & 1 & 1 & 1 \\
\hline 34 & A 35 & 2 & 2 & 3 & 1 & 3 & 3 & 2 & 2 & 1 & 1 \\
\hline 35 & A 36 & 3 & 3 & 1 & 2 & 3 & 3 & 2 & 2 & 1 & 1 \\
\hline 36 & A 37 & 2 & 3 & 1 & 2 & 3 & 2 & 2 & 3 & 2 & 2 \\
\hline 37 & A 38 & 2 & 2 & 1 & 1 & 2 & 2 & 2 & 2 & 1 & 1 \\
\hline 38 & A 39 & 2 & 2 & 1 & 1 & 3 & 2 & 2 & 2 & 1 & 1 \\
\hline 39 & A 40 & 2 & 2 & 1 & 2 & 2 & 2 & 2 & 2 & 0 & 0 \\
\hline 40 & A 41 & 2 & 3 & 1 & 2 & 2 & 3 & 2 & 2 & 1 & 1 \\
\hline 41 & D 63 & 0 & 2 & 1 & 2 & 2 & 2 & 2 & 2 & 1 & 1 \\
\hline 42 & D 64 & 0 & 1 & 2 & 2 & 3 & 2 & 3 & 3 & 2 & 2 \\
\hline 43 & D 74 & 2 & 2 & 3 & 2 & 2 & 2 & 2 & 1 & 2 & 2 \\
\hline
\end{tabular}


component values for each plant were measured in two dimensions. For each character, the PCA was employed to assess the distinctions between species, to decide how well the morphology helps to separate groups from each other, and to recognize any morphological discontinuities or overlaps within and between the Abutilon species taxa under study. PCA tests were performed using Minitab package 15.1.30 [27].

\section{Results}

\subsection{Morphological Characters}

Growth Habit. The habit of the species under study was defer from small shrub about $0.8 \mathrm{~m}$ high to large shrub up $3.7 \mathrm{~m}$ high. This character has divided the six species into three groups, first group include plants high ranging from meter or less, as in A. grandifolium and A. pannosum (Figure 1(A)), the second group contains species high form meter and less or equal to two and a half meters, as in A. grandifolum, A. pannosum, A. hirtum, A. figarianum, A. fruticosum and $A$. bidentatum (Figure $1(\mathrm{~B})$ ) and the third group compress species that range greater than two and a half meters, as in $A$. pannosum (Figure 1(C)).

Colour of Plant: The main colour of Abutilon plants are green as in A. hirtum, A. figarianum, A. grandifolum, A. pannosum (Figure 2(A)), greyish green as in

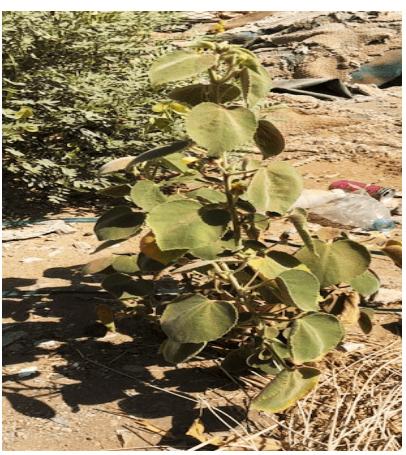

(A)

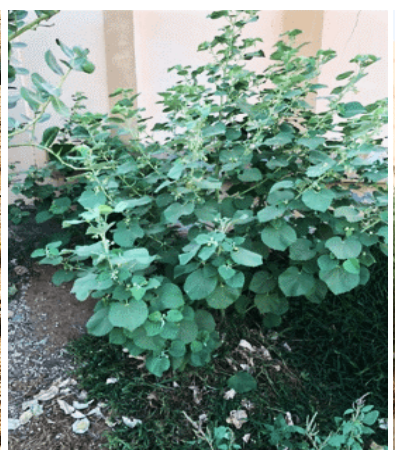

(B)

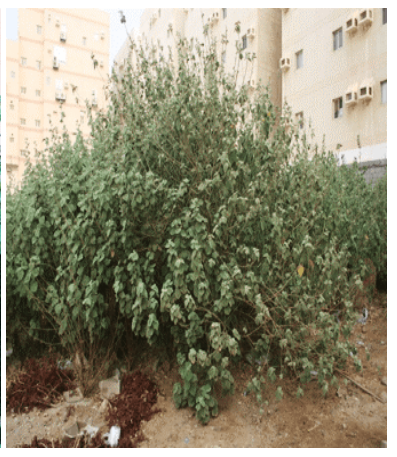

(C)

Figure 1. Shows different habit of Abutilon plants, (A) Shrub $\leq 1 \mathrm{~m}$; (B) Shrub $>1 \mathrm{~m} \leq 2.5$ $\mathrm{m}$; (C) Shrub > $2.5 \mathrm{~m}$.

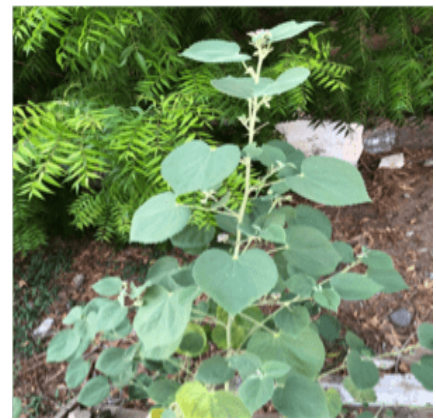

(A)

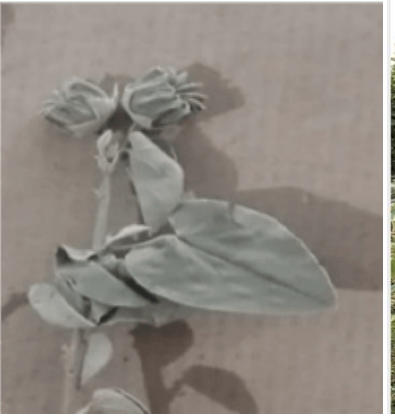

(B)

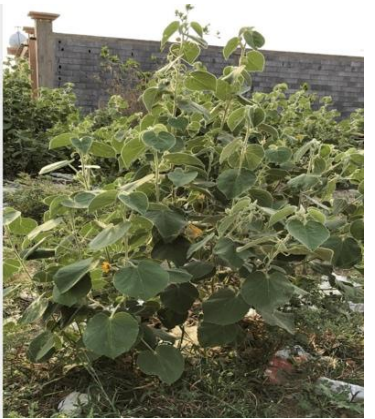

(C)

Figure 2. Shows different colour of Abutilon plants in the field: (A) Green colour, (B) Greyish green, (C) Yellowish green. 
A. fruticosum (Figure 2(B)) and yellowish-green as in A. bidentatum, A. figarianum and $A$. pannosum (Figure 2(C)).

\subsection{Leaf Characters}

Leaf Shape: there are two type of leaf shape in the six studding Abutilon species. The common shape is cordate as in $A$. grandifolium and $A$. pannosum (Figure 3(A)), the second shape is ovate to broadly ovate as in A. fruticosum, $A$. bidentatum, A. hirtum, A. figarianum, A. grandifolium and A. pannosum (Figure 3(B)).

Leaf Surface: plants of $A$. fruticosum species have glabrous leaf surface (Figure 4(A)), while leaves are velvety in $A$. hirtum and $A$. pannosum (Figure 4(B)), pubescent to tomentose as in $A$. bidentatum, $A$. figarianum and $A$. pannosum (Figure 4(C)) and the leaves are hairy surface in $A$. grandifolium (Figure 4(D)).

Leaf Apex. Several shapes for leaf apex were discovered in the six Abutilon species. Acute apex as in $A$. figarianum and $A$. pannosum (Figure 5(A)), cuspidate apex as in $A$. bidentatum, $A$. figarianum and $A$. grandifolium (Figure 5(B)), acute to cuspidate apex as in $A$. fruticosum $A$. hirtum, $A$. figarianum and $A$. pannosum (Figure 5(C)) and obtuse apex as in $A$. pannosum (Figure 5(D)).

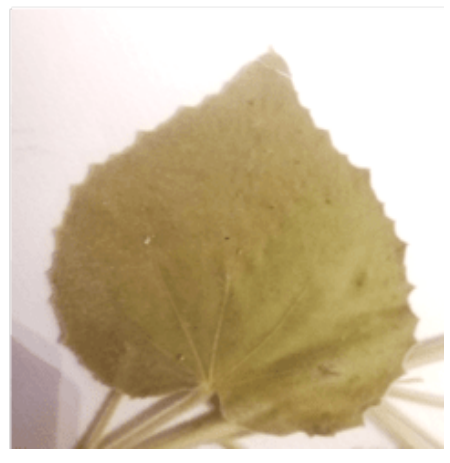

(A)

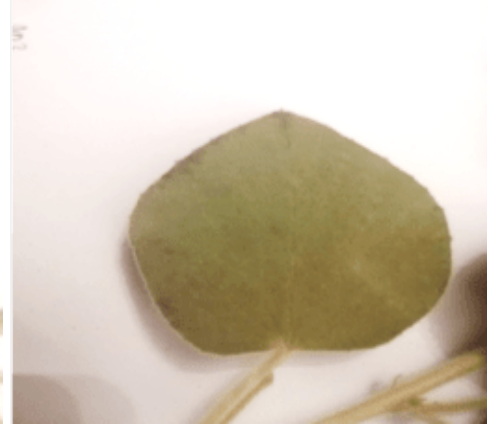

(B)

Figure 3. Shows two shapes of leaves, (A) Cordate, (B) Ovate to broadly ovate.

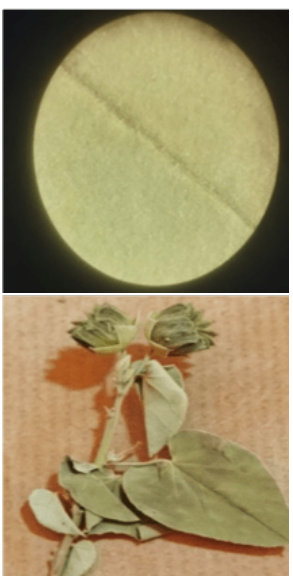

(A)

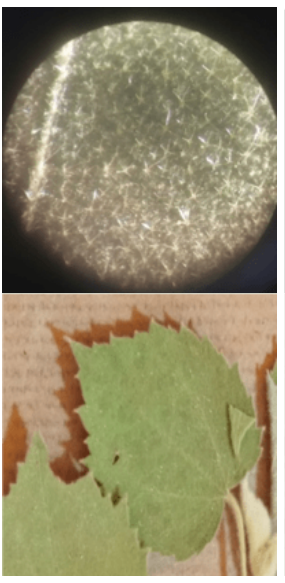

(B)

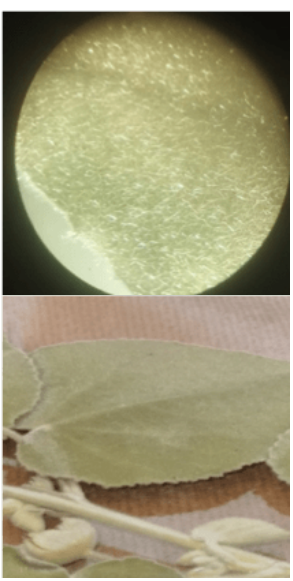

(C)

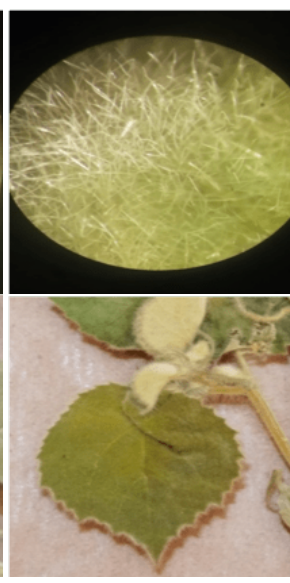

(D)

Figure 4. Shows variations in the leaf surface, (A) Glabrous, (B) Velvety, (C) Pubescent to tomentose, (D) Hairy. 


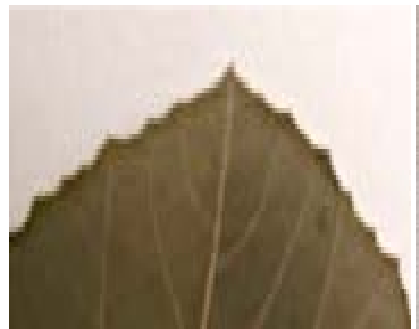

(A)

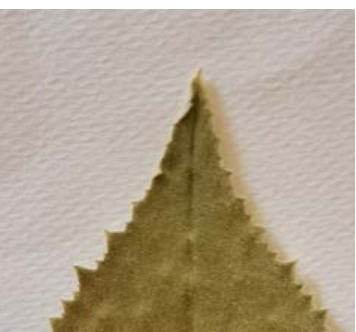

(B)

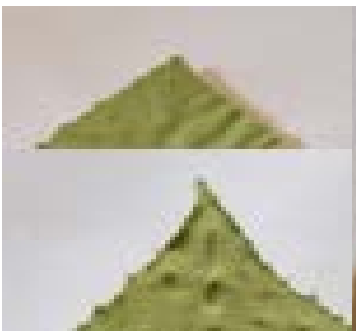

(C)

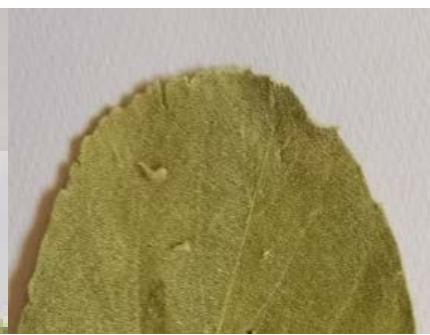

(D)

Figure 5. Shows four leaf apex shapes, (A) Acute, (B) Cuspidate, (C) Acute to cuspidate, (D) Obtuse.

Leaf Base: three types of leaf base were observed in the six Abutilon species, the cordate leaf base as in the $A$. fruticosum, $A$. bidentatum, A. hirtum, A. figarianum, $A$. grandifolium and $A$. pannosum species (Figure 6(A)), truncate leaf base as in the $A$. pannosum species (Figure 6(B)) and cordate to truncate leaf base were recorded in the $A$. figarianum and the $A$. pannosum species (Figure $6(\mathrm{C}))$.

Leaf Margin: There are three types of leaf margin shapes were observed in the studying Abutilon species. Double serrate margin in A. pannosum (Figure 7(A)), double denticulate to dentate leaf margin as in $A$. bidentatum, $A$. hirtum and $A$. figarianum (Figure $7(\mathrm{~B})$ ) and entire leaf margin as in the $A$. fruticosum A. grandifolium and $A$. pannosum species (Figure $7(\mathrm{C})$ ).

\subsection{Flower Characters}

Colour of Petal: the colour of petal in the six Abutilon species is differ. Flowers of some species have yellow colour such as $A$. bidentatum, A. figarianum and $A$. grandifolium (Figure 8(A)), yellow pale flower as in the $A$. fruticosum and $A$. hirtum species (Figure $8(\mathrm{~B})$ ) and yellow with red in the centre as in the plants of A. pannosum species (Figure $8(\mathrm{C})$ ).

\subsection{Fruit Characters}

Fruit Shape: there are two types of shape that can distinguish the six Abutilon species; globose shape as in $A$. figarianum and $A$. pannosum species (Figure 9(A)), and cylindric shape as in the $A$. fruticosum, $A$. bidentatum, $A$. hirtum and $A$. grandifolium (Figure 9(B)).

Carpel Shape: This study presented two different shapes of carpel, the first shape is a reniform with a rounded apex as in $A$. figarianum and $A$. pannosum species (Figure $10(\mathrm{~A})$ ), while the second shape is a reniform with pointed apex as in the $A$. fruticosum, $A$. hirtum, $A$. bidentatum and $A$. grandifolium species (Figure 10(B)).

\subsection{Morphometric Analysis}

Cluster Analysis (UPGMA): The UPGMA analysis separated the six Abutilon species into three major clusters (Figure 11). Cluster I divided into two subclades, first subclade includes three samples of $A$. grandifolium, while the second 


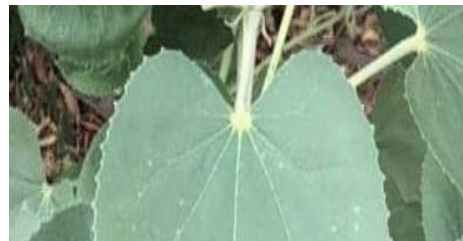

(A)

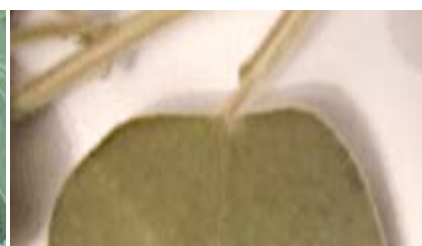

(B)

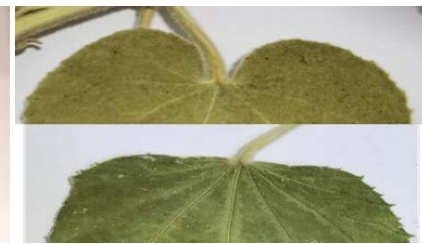

(C)

Figure 6. Shows different types of leaf base, (A) Cordate, (B) Truncate, (C) Cordate to truncate.

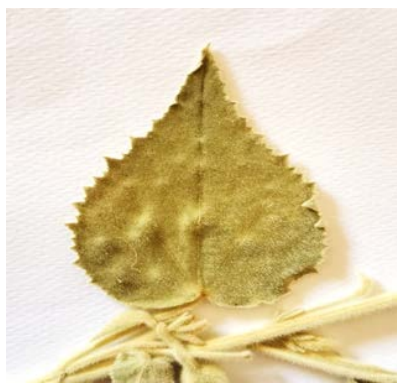

(A)

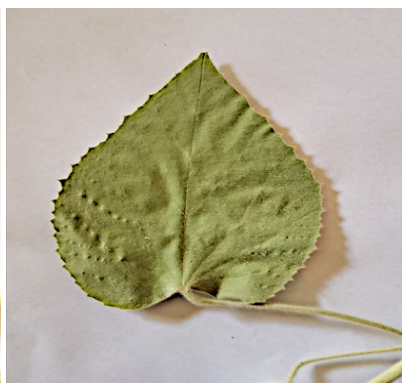

(B)

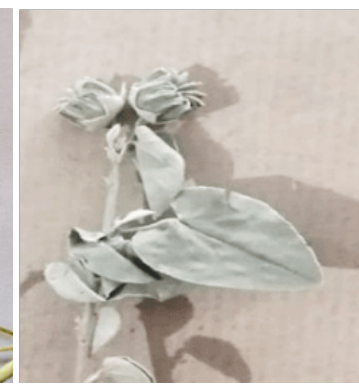

(C)

Figure 7. Shows different shapes of the leaf margin of the studied Abutilon species, (A) Double serrate, (B) Double denticulate to dentate, (C) Entire.

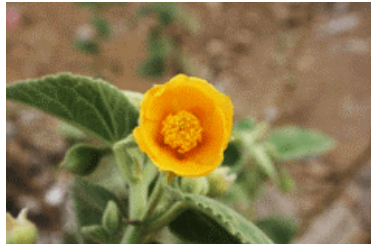

(A)

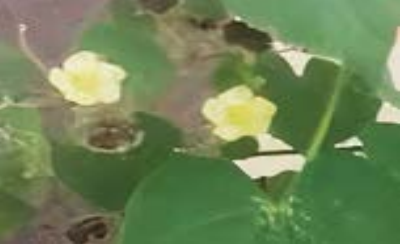

(B)

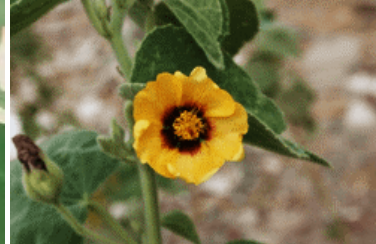

(C)

Figure 8. Shows different petal colours in Abutilon species: (A) Yellow, (B) Yellow pale, (C) Yellow with red centre.

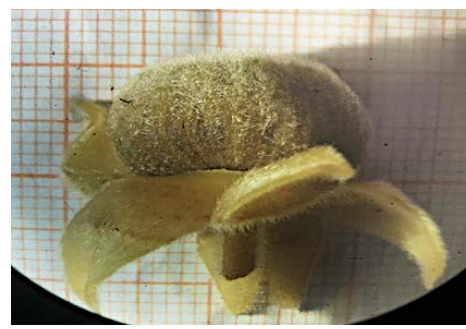

(A)

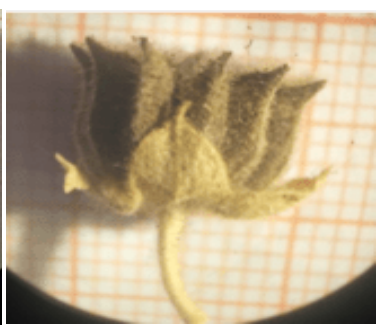

(B)

Figure 9. Shows different shapes of fruits in Abutilon species: (A) Globose, (B) Cylindric.

subclade contains three samples of $A$. figarianum. Cluster II divided into two subclades, first clade includes one samples of $A$. hirtum and whereas the second clade includes $A$. bidentatum, and $A$. fruticosum. Cluster III includes samples of the $A$. pannosum species.

Principle Coordinate Analysis (PCoA): Based on morphological data, the Gower's general similarity coefficient (Gower, 1966) was used for first three axis in the PCoA (Figure 12(A) and Figure 12(B)). The first three principle coordinate 


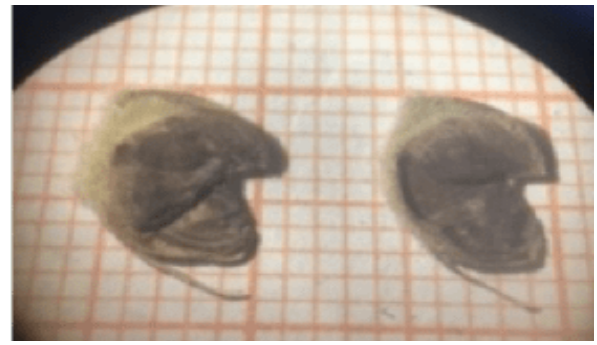

(A)

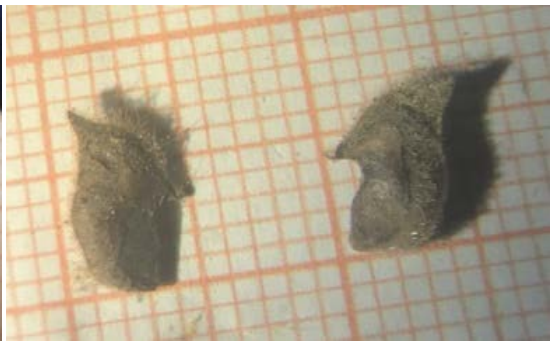

(B)

Figure 10. Shows carpel shapes of Abutilon species: (A) Reniform with rounded apex; (B) Reniform with pointed apex.

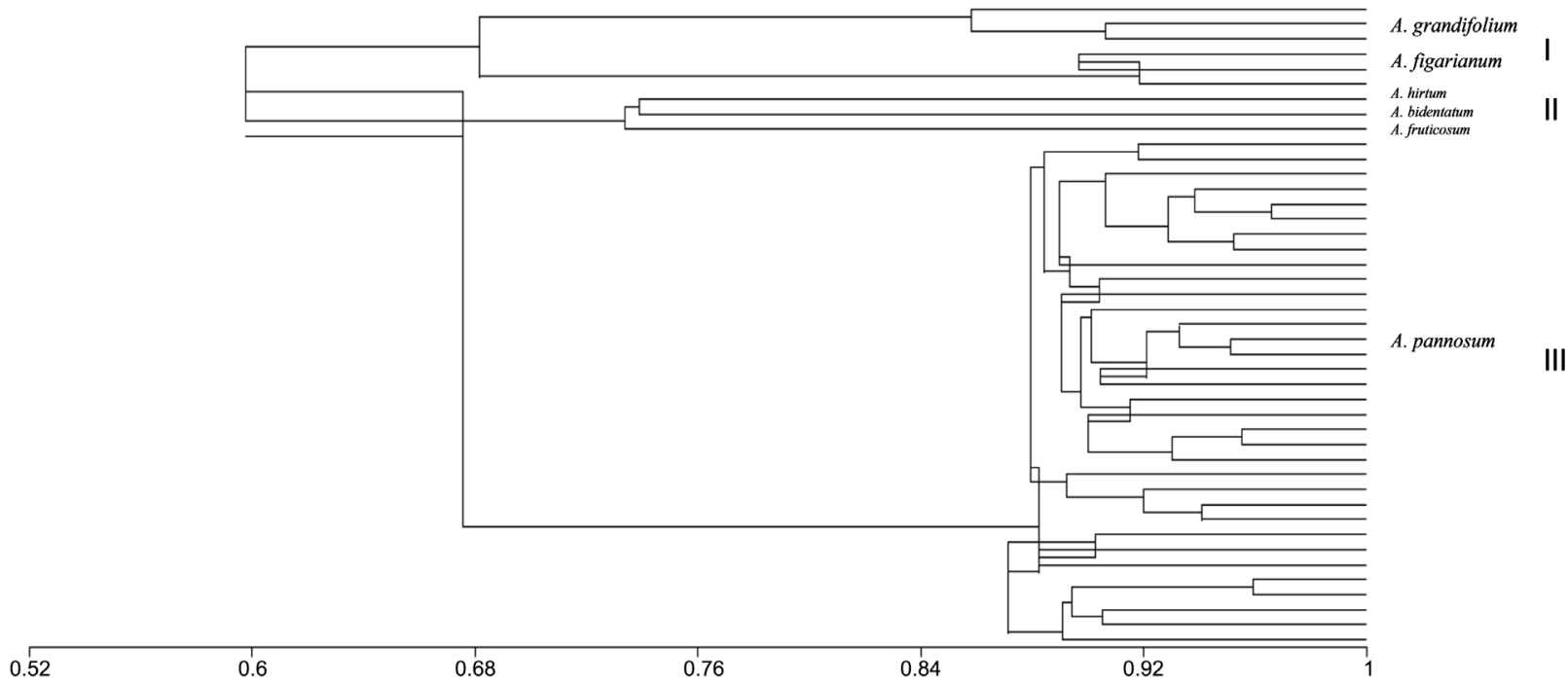

Figure 11. The UPGMA analysis representation of morphological data of 43 accessions of Abutilon species. Roman numerals indicate clades number.

axis accounted for axis 1 (24.996\%), axis $2(12.835 \%)$ and axis 3 (7.562\%). The variance of the first and second principle coordinate accounted $37.831 \%$ and the first and third principle coordinate accounted $32.558 \%$ of the total variation and the ordination of these three-axis showed six groups (Figure 12(A) and Figure 12(B)). Group 1 includes three samples of $A$. grandifolium, group 2 includes three samples of $A$. figarianum, group 3 includes one sample of $A$. hirtum, group 4 includes one sample of $A$. bidentatum, group 5 includes one sample of $A$. fruticosum, group 6 includes 34 samples of A. pannosum species (Figure 12(A) and Figure 12(B)).

Principle Component Analysis (PCA): The patterns variations of the quantitative morphological characters are represented in the first two axis in the PCA score plot (Figure 13). The result of the PCA showed that the plants of the six Abutilon species under this study are separated into six groups (Figure 13). The Loading plot (Figure 14) is used for investigating the importance of each variable to each component and to define the eigenvalue of each axis (Table 6). A scree plot is useful to get information on the importance of the eigenvalues that are represented by PCA (Figure 15). 

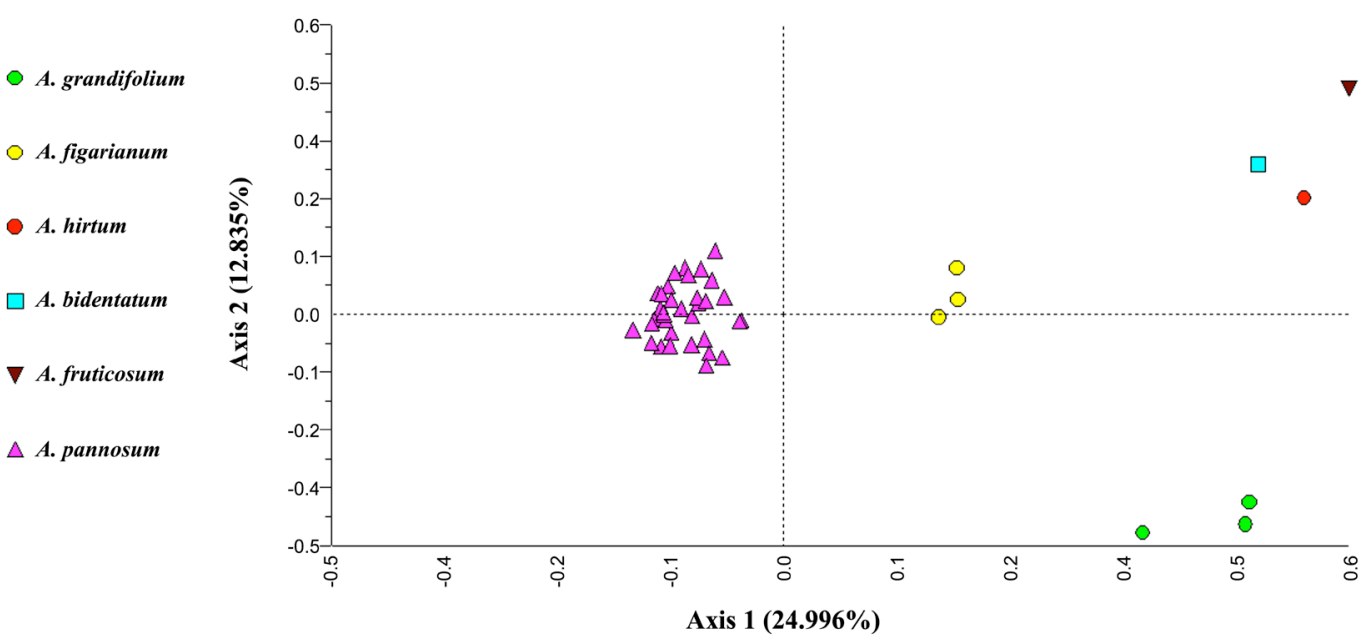

(A)
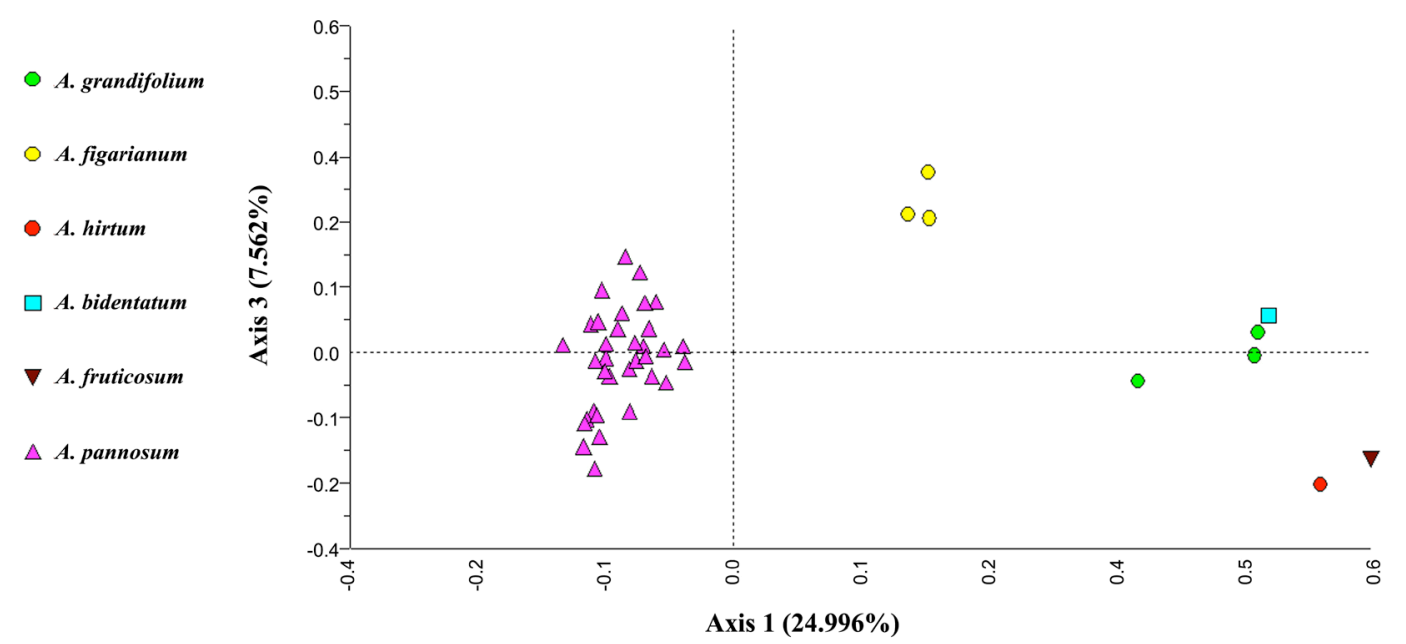

(B)

Figure 12. The PCoA representation of morphological data of 43 samples of the six Abutilon species. (A) Principle coordinate axis 1 and 2. (B) Principle coordinate axis 1 and 3.

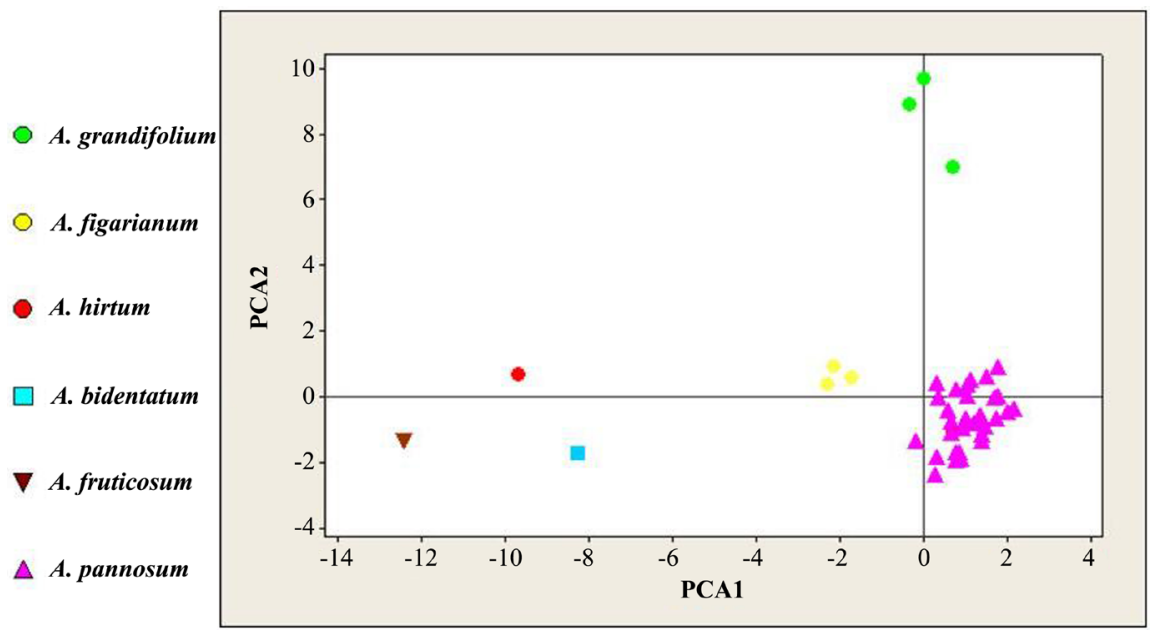

Figure 13. Principle component axis 1 and 2 represent of morphological data of $43 A b$ utilon species. 


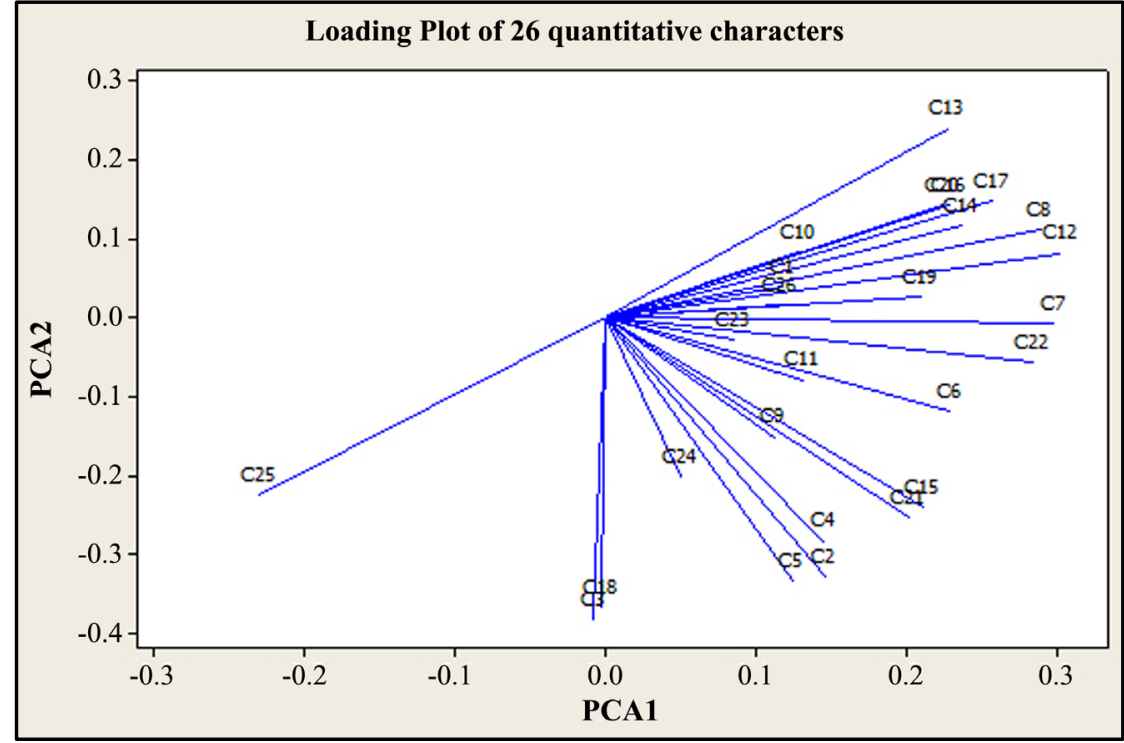

Figure 14. Loading plot of PCA for 26 quantitative morphological characters of $43 A b$ utilon species.

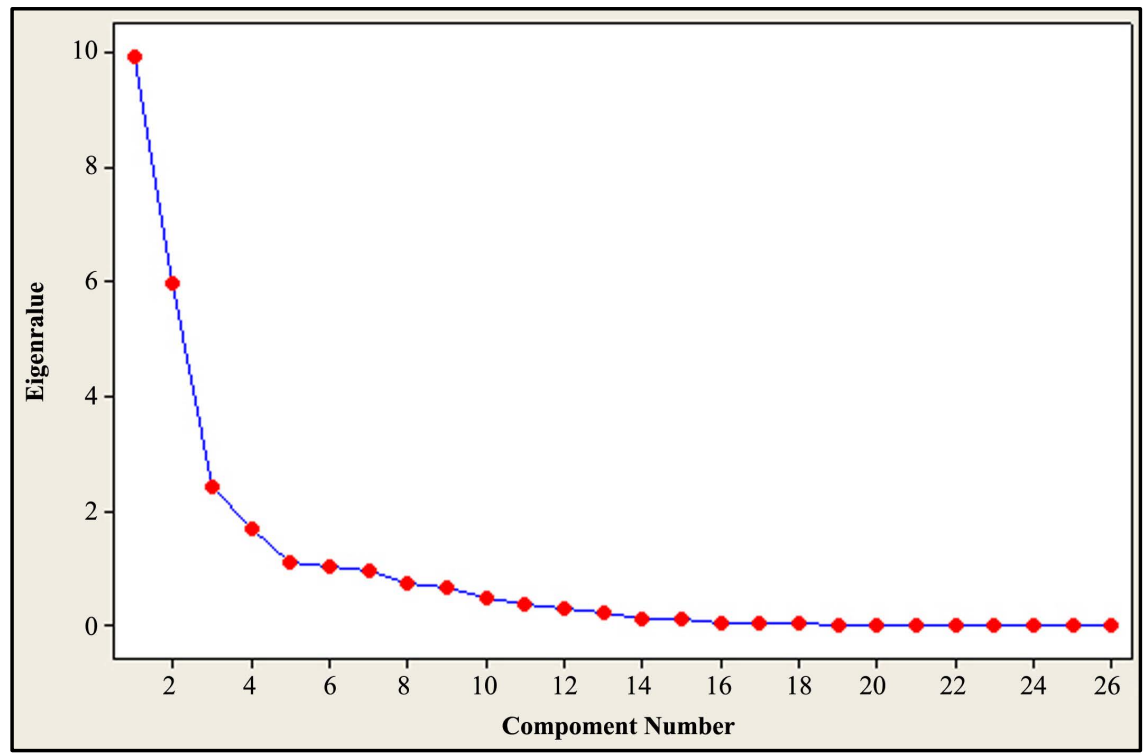

Figure 15. Scree plot of PCA for the 26 quantitative morphological characters of Abutilon species.

Table 6. Characters description and character abbreviation, loading on principal axis and Eigen analysis of principle component analysis of 43 plants of Saudi Arabian Abutilon species based on 26 quantitative morphological characters.

\begin{tabular}{lcccccc}
\hline Morphological characters and character abbreviation & PC1 & PC2 & PC3 & Eigenvalue & Proportion & Cumulative \\
\hline C1: Plant high $(\mathrm{m})$ & 0.118 & 0.038 & 0.022 & 9.9289 & 0.382 & 0.382 \\
C2: Length of stipulate (mm) & 0.146 & -0.328 & -0.116 & 5.9846 & 0.23 & 0.612 \\
C3: Length of petiolate (cm) & -0.007 & -0.381 & 0.067 & 2.4129 & 0.093 & 0.705 \\
C4: Length of midrib (cm) & 0.146 & -0.283 & 0.146 & 1.6646 & 0.064 & 0.769 \\
C5: Length of leaf $(\mathrm{cm})$ & 0.125 & -0.332 & 0.128 & 1.0805 & 0.042 & 0.81 \\
\hline
\end{tabular}




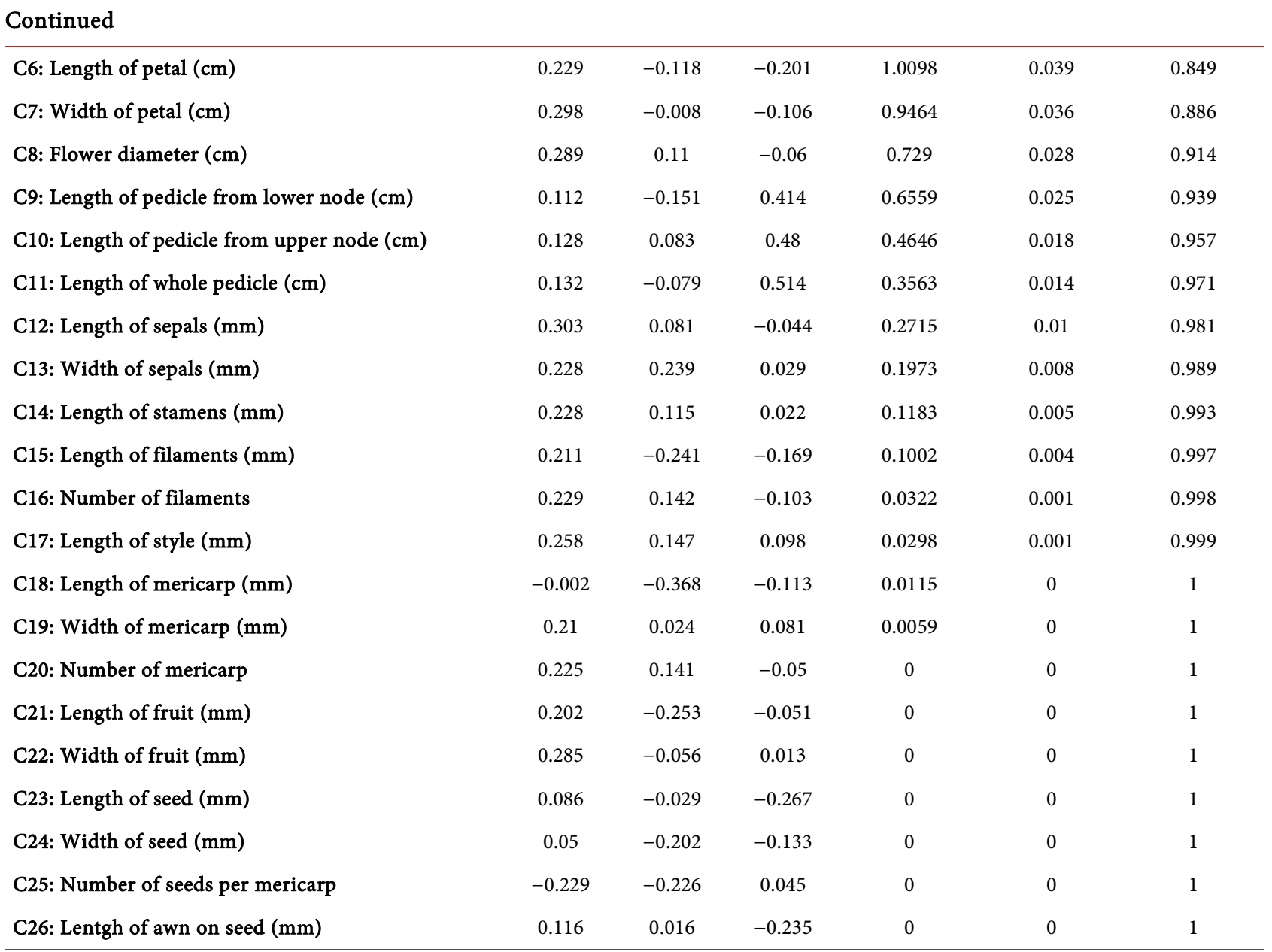

\section{Discussion}

Some morphological characters can be used in the field to differentiate Abutilon species from each other such as: habit, plant colour, leaf structures, flower colour and fruit features. This study distinguished six species of Abutilon ( $A$. fruticosum, A. bidentatum, A. hirtum, A. figarianum, A. grandifolium and A. Pannosum) that were collected from different locations of Saudi Arabia based on morphological and morphometric data.

The $\boldsymbol{A}$. fruticosum species is well known in Saudi Arabia and were reported by previous authors (Collenette 1985, 1999; Migahid 1996; Chaudhary 2001; Taia 2009). Collenette (1999) cited two taxa affinities to A. fruticosum species and she called one $A$. sp. aff. fruticosum and the second $A$. sp. nov. aff. fruticosum. However, our study's findings did not agree with these taxa affinities. Plants of this species are distributed in the central and from north-western to southwestern of Saudi Arabia (Table 1 and Figure 16) and they are distinguished by several characters, colour of leaf is grayish green (Figure 2(B)), leaf shape is ovate to broadly ovate (Figure 3(B)), leaf surface is glabrous (Figure 4(A)), leaf apex is acute to cuspidate (Figure $5(\mathrm{C})$ ), leaf base is cordate (Figure 6(A)), leaf margin is entire (Figure $7(C)$ ), colour of flower is yellow pale (Figure $8(B)$ ), fruit 


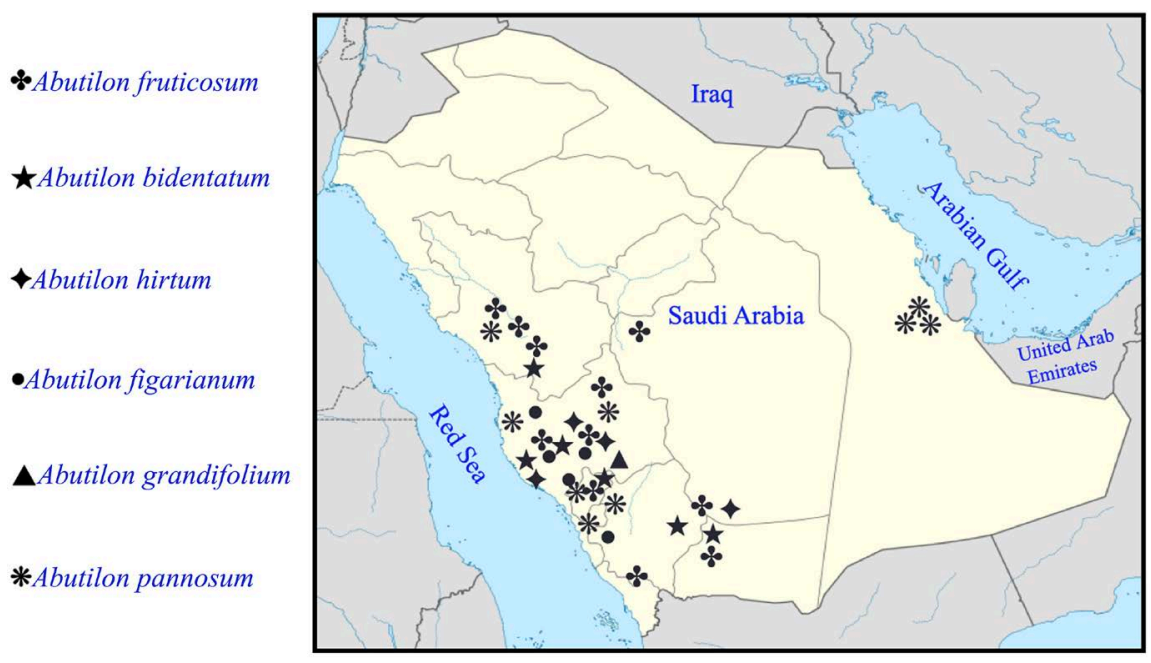

Figure 16. A distribution map of Abutilon species in Saudi Arabia.

shape is cylindric (Figure $9(B)$ ), the mericarp is reniform with pointed apex (Figure 10(B)).

Species of $\boldsymbol{A}$. bidentatum is also well known in Saudi Arabia and was recorded by previous authors (Collenette 1985, 1999; Migahid 1996; Chaudhary 2001; Taia 2009). Collenette (1999) reported two taxa affinity to $A$. bidentatum species and she called one $A$. sp. aff. bidentatum and the second is $A$. sp. nov. aff. bidentatum. However, our study's findings did not agree with these taxa affinities. Plants of this species are distributed in the western to southern regions of Saudi Arabia (Table 1 and Figure 16) and they are distinguished by several characters, colour of leaf is yellowish green (Figure 2(C)), leaf shape is ovate to broadly ovate (Figure 3(B)), leaf surface is pubescent to tomentosa (Figure $4(\mathrm{C})$ ), leaf apex is cuspidate (Figure 5(B)), leaf base is cordate (Figure 6(A)), leaf margin is double denticulate to dentate (Figure 7(B)), colour of flower is yellow (Figure $8(\mathrm{~A})$ ), fruit shape is cylindric (Figure $9(\mathrm{~B})$ ), the mericarp is reniform with pointed apex (Figure 10(B)).

Species of $\boldsymbol{A}$. hirtum was recorded by previous authors such as: Collenette 1999; Migahid 1996; Chaudhary 2001; Taia 2009. Plants of this species are distributed western and in the southern region of Saudi Arabia (Table 5 and Figure 16) and they are distinguished by several characters, colour of leaf is green (Figure 2(A)), leaf shape is ovate to broadly ovate (Figure 3(B)) leaf surface is velvety (Figure $4(B)$ ), leaf apex is acute to cuspidate (Figure $5(C)$ ), leaf base is cordate (Figure 6(A)), leaf margin is double denticulate to dentate (Figure $7(B)$ ), colour of flower is yellow pale (Figure $8(B)$ ), fruit shape is cylindric (Figure 9(B)), the mericarp is reniform with pointed apex (Figure 10(B)).

Species of $\boldsymbol{A}$. figarianum was recorded by previous authors such as: Collenette 1999; Migahid 1996; Chaudhary 2001. Taia (2009) cited incorrect scientific name of $A$. figarium, the correct scientific name is $A$. figarianum. Plants of this species are distributed in the southern and western to southwestern regions of Saudi Arabia (Table 1 and Figure 16) and they are distinguished by several 
characters, colour of leaf is green (Figure 2(A)) but sometimes in some samples yellowish green (Figure $2(\mathrm{C})$ ), leaf shape is ovate to broadly ovate (Figure $3(B)$ ), leaf surface is pubescent to tomentosa (Figure $4(C)$ ), leaf apex is cuspidate (Figure 5(B)), or sometimes acute (Figure 5(A)) or acute to cuspidate (Figure 5(C)), leaf base is cordate to truncate (Figure 6(C)) sometimes cordate (Figure 6(A)), leaf margin is double denticulate to dentate (Figure $7(B)$ ), colour of flower is yellow (Figure 8(A)), fruit shape is globose (Figure $9(\mathrm{~A})$ ) and the mericarp is reniform with rounded apex (Figure 10(A)).

The species of $\boldsymbol{A}$. grandifolium was previously reported by Chaudhary (2001) only. Collentte $(1985,1999)$ nor Taia (2009) recorded this species distributed in Saudi Arabia. Plants of this species are distributed in the western region (Table 1 and Figure 16) and they are distinguished by several characters, colour of leaf is green (Figure 2(A)), leaf shape is ovate to broadly ovate (Figure 3(B)) in some specimen are cordate (Figure 3(B)), leaf surface is hairy (Figure 4(D)), leaf apex is cuspidate (Figure 5(B)), leaf base is cordate (Figure 6(A)), leaf margin is double denticulate to dentate (Figure 7(B)), colour of flower is yellow (Figure $8(\mathrm{~A})$ ), fruit shape is cylindric (Figure $9(\mathrm{~B})$ ) and the mericarp is reniform with pointed apex (Figure 10(B)).

The species of $\boldsymbol{A}$. pannosum is well known in Saudi Arabia and was reported by previous authors (Collenette 1999; Migahid 1996; Chaudhary 2001; Taia 2009). Plants of this species are widespread in Saudi Arabia, from north-western to southwestern of the country and in the eastern region (Table 1 and Figure 16) and they are distinguished by several characters, there is a variation of leaf formation in this species. Its Colour of leaf is green (Figure 2(A)) sometimes yellowish green (Figure 2(C)), leaf shape is ovate to broadly ovate (Figure 3(B)) sometimes in some specimens is cordate (Figure $3(\mathrm{~A})$ ), leaf surface is pubescent to tomentosa (Figure 4(C)) but sometimes is velvety (Figure 4(B)), leaf apex is differ, it can be cuspidate (Figure 5(B)) or acute to cuspidate (Figure 5(C)) obtuse (Figure 5(D)) and sometimes acute (Figure 5(A)), leaf base is also differ, cordate (Figure 6(A)) or truncate (Figure $6(\mathrm{~B})$ ) and cordate to truncate (Figure $6(C)$ ), leaf margin is double denticulate to dentate (Figure $7(B)$ ) and sometime double serrate (Figure $7(\mathrm{~A})$ ), colour of flower is consistent color, its yellow with red center (Figure $8(C)$ ), and the fruit shape is globose (Figure $9(\mathrm{~A})$ ), the mericarp is reniform with rounded apex (Figure 10(B)).

The numerical taxonomy based on the morphological characters including quantitative and qualitative in the PCoA and the UPGMA analysis and based on quantitative characters in the PCO analysis indicated that the Abutilon plants under this study distinguished into six groups with realistic agreement in all analysis results. The results of the UPGMA analysis (Figure 11) separated all 43 examined samples into three main clusters. First cluster includes all plants of $A$. grandifolium and A. figarianum, second cluster includes all plants of $A$. hirtum, $A$. bidentatum and $A$. fruticosum, while all 34 plants of the $A$. pannosum species are located in the third cluster. In the PCoA based on 26 quantitative and 10 qualitative characters (Figure 12(A) and Figure 12(B)) and PCA analyses based on 
only 26 quantitative morphological characters (Figure 13) of the Abutilon accessions are clearly separated into six main groups in the axis one and three (Figure 12(A) and Figure 12(B); Figure 13). The group 1 includes three samples of $A$. grandifolium species, likewise, group 2 includes three samples of $A$. figarianum species, while only one sample of $A$. hirtum in group 3, $A$. bidentatum in group $4, A$. fruticosum in group 5 and 34 samples of $A$. pannosum species gathered in group 7 (Figure 12(A) and Figure 12(B); Figure 13).

\section{Conclusion}

This current study provides the morphological characteristics which are effective in Abutilon species identification. Six species of genus Abutilon were distinguished and identified using morphological characteristics and morphometric analysis of the PCoA, the UPGMA and the PCA.

\section{Statement of Ethics}

Ethical approval is not required for this type of research.

\section{Funding Sources}

The authors received no financial support for the research.

\section{Acknowledgements}

Thanks to Maryam Alshebramy, Salehm Algharbi, Amer Alasiri, Muhammed Althawabi, Muhammed Almalki, Muhammed Alshawi for their help during field trips.

\section{Conflicts of Interest}

The authors declared no conflict of interest.

\section{References}

[1] Christenhusz, M.J.M. and Byng, J.W. (2016) The Number of Known Plants Species in the World and Its Annual Increase. Phytotaxa, 261, 201-217. https://doi.org/10.11646/phytotaxa.261.3.1

[2] Taia, W.K. (2009) General View of Malvaceae Juss. S.L. and Taxonomic Revision of Genus Abutilon Mill. In Saudi Arabia. Journal of King Abdulaziz University, Science, 21, 349-363. https://doi.org/10.4197/Sci.21-2.12

[3] Gomaa, A.A., Samy, M.N., Desoukey, S.Y. and. Kamel, M.S. (2016) Pharmacognostical Studies of Leaf, Stem, Root and Flower of Abutilon hirtum (Lam.) Sweet. In ternational Journal of Pharmacognosy and Phytochemical Research, 8, 199-216.

[4] Bentham, G. and Hooker, J.D. (1862) Genera Plantarum. L. vol. 1, Reeve and Co., London.

[5] Hutchinson, J. (1967) The Genera of Flowering Plants (Angiospermoe). Vol. 2, Clarendon Press, Oxford.

[6] Fryxell, P.A. (1988) Malvaceae of Mexico. Systematic Botany Monographs, 25, 1-522. https://doi.org/10.2307/25027717 
[7] Takhtajan, A. (2009) Flowering Plants. Springer Verlag, Berlin. https://doi.org/10.1007/978-1-4020-9609-9

[8] Reveal, J.L. (2012) An Outline of a Classification Scheme for Extant Flowering Plants. Phytoneuron, 37, 1-221.

[9] Baker, E.G. (1893) Abutilon. In Synopsis of Genera and Species of Malveae. The Journal of Botany, 31, 71-76.

[10] Mattei, G.E. (1915) Studi Sugli Abutilon dell'Africa Orientale. Bollettino delle Reale orto Botanico di Palermo, 1, 69-102.

[11] Husain, S.A. and Baquar, S.R. (1974) Biosystematic Studies in the Genus Abutilon from Pakistan. Pakistan Council of Scientific and Industrial Research, 15, 219-234.

[12] Mabberley, D.J. (1987) The Plant Book, a Portable Dictionary of the Higher Plants. Cambridge University Press, New York.

[13] Fryxell, P.A. (1997) The American Genera of Malvaceae-II. Brittonia, 49, 204-269. https://doi.org/10.2307/2807683

[14] El Naggar, S.M. (2004) Pollen Morphology of Egyptian Malvaceae: An Assessment of Taxonomic Value. Turkish Journal Botany, 28, 227-240.

[15] Shaheen, N., Khan, M.A., Hayat, M.G. and Yasmin, G. (2009) Pollen Morphology of 14 Species of Abutilon and Hibiscus of the Family Malvaceae (Sensu Stricto). Journal of Medicinal Plants Research, 3, 921-929.

[16] Chaudhary, S.A. (2001) Flora of the Kingdom of Saudi Arabia. Vol. 3, Ministry of Agriculture and Water, Riyadh.

[17] Alfarhan, A.H., Al-Turki, T.A. and Basahy, A.Y. (2005) Flora of Jizan Region. Vol. 1, King Abdulaziz City for Science and Technology, Riyadh.

[18] Gill, A. and Kaur, R. (2015) Cytomorphological Investigations of Some Species of Abutilon Mill. from Punjab. Journal on New Biological Reports, 4, 219-227.

[19] Shaheen, H. (2015) Floristic and Ethnobotanical Enumeration of the Desert, Punjab, Pakistan. Doctoral Dissertation, Department of Botany, Faculty of Sciences, Arid Agriculture University Rawalpindi, Pakistan.

[20] Bano, I. and Deora, G.S. (2017) Studies on Micromorphological Taxonomic Variations in Abutilon Species of Indian Thar Desert. IOSR Journal of Pharmacy and Biological Sciences, 60-68.

[21] Migahid, A.M. (1988) Flora of Saudi Arabia. Vol. 1, Riyadh University Publications, Riyadh, Saudi Arabia.

[22] Anatriello, L. (2007) Abutilon Plant Named 'ATN YB5'. United States Plant Patents. Washington, DC, Patent and Trademark Office, 17-77.

[23] Kunnur, S. B and Kkotresha, K. (2011). Abutilon ramousum (CAV.) \& Gill \& Perr. [Malvaceae]: A new record for Karnataka state. Journal of Economic and Taxonomic Botany, pp. 215-217.

[24] Brown, G.N. (2012) Mandevilla Plant Named 'ALEGNUFLORA709'. United States Plant Patent. Washington, DC, Patent and Trademark Office, 17-771.

[25] Gower, J.C. (1966) Some Distance Properties of Latent Root and Vector Methods Used in Multivariate Analysis. Biometrika, 53, 326-338.

https://doi.org/10.1093/biomet/53.3-4.325

[26] Kovach, W.L. (1999) MVSP - A Multivariate Statistical Package for Windows, Version 3.1. Kovach Computing Services, Pentraeth.

[27] Ryan, B.F., Joiner, B.L. and Ryan, T.A. (1985) Minitab Handbook. PWS-KENT 
Publishing Company, Boston.

[28] Sneath, P.H. and Sokal, R.R. (1973) Numerical Taxonomy: The Principles and Practice of Numerical Classification. 1st Edition, W.H. Freeman, San Francisco. 